This is the final peer-reviewed accepted manuscript of:

R. Amorati, L. Valgimigli. Advantages and limitations of common testing methods for antioxidants. Free Radical Research, 2015, 49, 633-649

The final published version is available online at:

https://doi.org/10.3109/10715762.2014.996146

Rights / License:

The terms and conditions for the reuse of this version of the manuscript are specified in the publishing policy. For all terms of use and more information see the publisher's website.

This item was downloaded from IRIS Università di Bologna (https://cris.unibo.it/)

When citing, please refer to the published version. 


\title{
Advantages and limitations of common testing methods for antioxidants
}

\author{
Riccardo Amorati and Luca Valgimigli*
}

University of Bologna, Department of Chemistry “G. Ciamician”, Via S. Giacomo 11, 40126-

Bologna, Italy.

*Corresponding author. e-mail: luca.valgimigli@unibo.it; tel. +390512095683; fax +390512095688 


\begin{abstract}
Owing to the importance of antioxidants in the protection of both natural and man-made materials, a large variety of testing methods has been proposed and applied. These include methods based on inhibited autoxidation studies, which are better followed by monitoring the kinetics of oxygen consumption or that of formation of hydroperoxides, the primary oxidation products. Analytical determination of secondary oxidation products (e.g. carbonyl compounds) has also been used. The majority of testing methods, however, does not involve substrate autoxidation. They are based on the competitive bleaching of a probe (e.g. ORAC assay, $\beta$-carotene and crocine bleaching assays, luminol assay), on reaction with a different probe (e.g. spin-trapping and TOSC assay), or they are indirect methods based on the reduction of persistent radicals (e.g. galvinoxyl, DPPH and TEAC assays), or of inorganic oxidizing species (e.g. FRAP, CUPRAC and Folin-Ciocalteu assays). Yet other methods are specific for preventive antioxidants. The relevance, advantages and limitations of these methods are critically discussed stepping from their chemistry and the mechanisms of antioxidant activity. A variety of cell-based assays has also been proposed to investigate the biological activity of antioxidants. Their importance and critical aspects are discussed, along with arguments for the selection of the appropriate testing methods, according to the different needs.
\end{abstract}

Keywords. Autoxidation, kinetics, rate constant, assay, peroxyl radicals. 


\section{Introduction}

The oxidation of organic materials is the unavoidable consequence of their existence under an oxygen-rich atmosphere and it mainly occurs by a free-radical-mediated process called autoxidation [1-4]. This condition equates man-made materials like plastics, pharmaceuticals, processed food, or cosmetics to biomolecules in a living organism and the chemistry underlying the autoxidation of such different compounds bears amazing similarity [1,5]. In nature as in manufacture, the most effective and convenient way to protect molecules from oxygen reactivity is to use antioxidants [3]. As a consequence of their enormous importance in the protection of most materials, thousands of potential antioxidants were developed in the last century and the need for practical ways to study them has brought to the design and application of a variety of testing methods. From the second half of the 20th century, the growing awareness of the importance of radical-mediated reactions in biology [6], the clarification of the role of vitamin E [5] and vitamin C [7] and other dietary antioxidants $[4,8-10]$ in the protection of biomolecules and the theories relating aging and disease to radical-mediated oxidative stress [11-14] have represented (and still represent) another major impulse to the study of antioxidants. In several cases these studies aim at the rapid screening of a large number of molecules, or the testing of natural extracts of complex or unknown composition endowed with some potential "antioxidant activity". To adapt to the different testing needs, an impressively large array of rapid assays has been proposed and some of them has actually been implemented in commercial testing kits allowing "one-button analysis" of the antioxidant capacity. We anticipate that not all methods have the same soundness. Some are inappropriate, bringing to numerical values or ranking of antioxidant performance of no physical or chemical meaning $[4,15]$, let alone the ability to predict a biological effect in vivo [12]. Yet other methods, under appropriate settings, would bring to relevant and useful information, however they are often used with little consideration of the chemistry behind them and how it is influenced by the experimental conditions. As a consequence they often produce inconsistent or contradicting results $[4,16,17]$, for instance 
between testing methods, or between different labs nominally using the same method, clearly hampering their usefulness and misleading future research.

Aim of this review is not to provide a comprehensive listing of all the proposed methods to test antioxidants, but to focus on a representative variety of them, selected for their soundness or for their popularity, and critically discuss their advantages and limitations stepping from their chemistry. We will also provide elements to guide the selection of the most appropriate methods according to the different needs, based on the experience of our research group. The review will focus on "chemical" testing methods, being of more general use and better established, while a shorter discussion will be dedicated to "cell-based" assays, which have been the subject of other recent reviews [15,18-21]. We anticipate that his review uses a different approach with respect to other reviews in this area [22-24]. For instance, Liu [22] offers a broad selection of chemical testing methods and a detailed description of their chemistry; however, methods are presented in an acritical fashion, not discussing their soundness, advantages and limits and providing no guidance for method selection. The reviews by Huang et al. [23] and Prior et al. [24], instead, provide critical discussion; however, classification of methods is based on the involved reaction with antioxidants, either hydrogen-atom transfer (HAT) or electron transfer (ET or SET), which appears to need revision in the light of the now deeper understanding of the mechanisms of reaction of antioxidants $[2,3]$. Their guidance for method selection gives no attention to reaction kinetics, which is the main focus of our discussion.

\section{What is an antioxidant?}

Possibly the major source of confusion in antioxidants testing arises from the uncertain definition of what an antioxidant is and what it is expected to do. In keeping with the pioneering work of Keith Ingold [1,5], we define an antioxidant as a substance (small molecule or complex system) that, when added to an oxidizable molecule in small amount (for small molecules, usually $<1 \%$ compared to the material to protect) is able to protect such molecule by delaying, retarding or 
inhibiting its autoxidation [2,4]. Although this definition is of general applicability for any molecule in solution, it also holds for biological systems and living organisms and is in accordance with the definition given by Halliwell and Gutteridge in this context [6]. Classification of the antioxidants is based on their mechanism of protection and is very important in order to set or select the most appropriate testing method.

Oxidizable molecules are most commonly hydrocarbons, or hydrocarbon moieties, both saturated and unsaturated, bearing other functional groups. In biological systems they are represented mainly by lipids (e.g. triglycerides and cholesterol), proteins and carbohydrates. Linoleic acid is arguably the most investigated and most representative oxidizable substrate and its autoxidation can be taken as a model to classify antioxidants (scheme 1) [2]. Autoxidation is initiated by some radical species $\mathrm{X} \bullet$ able to react with the substrate $\mathrm{RH}$ (most commonly by $\mathrm{H}$-atom abstraction) to yield an alkyl radical $\mathrm{R} \bullet$, which will react at diffusion-controlled rate with oxygen to form a peroxyl radical (ROO•). The peroxyl radical ROO• attacks another molecule of the substrate to yield a hydroperoxide $\mathrm{ROOH}$ (the oxidized substrate) and another radical, thereby establishing the chainreaction. The chain reaction proceeds for many cycles (propagation) before two radicals incidentally quench each other (termination step) [1,2].

\section{<scheme 1 here>}

Compounds able to impair this radical chain reaction are called direct antioxidants and are divided into two main groups. Preventive antioxidants interfere with the initiation process [2]. Examples of such are the enzyme catalase [EC 1.11.1.6] and metal chelators such as phytic acid. One of the main sources of initiation is the Fenton reaction (or similar) occurring between the reduced state of transition metal ions $\left(\right.$ e.g. $\left.\mathrm{Fe}^{2+}\right)$ and hydrogen peroxide $(\mathrm{HOOH})$ or organic hydroperoxides (ROOH) yielding hydroxyl or hydroxyl and alcoxyl radicals (eq. 1), which initiate the chain.

$$
\mathrm{H}_{2} \mathrm{O}_{2}+\mathrm{Fe}^{2+} \longrightarrow \mathrm{HO} \cdot+\mathrm{HO}^{-}+\mathrm{Fe}^{3+}
$$


By blocking redox active metal ions in an oxidized form $\left(e . g . \mathrm{Fe}^{3+}\right)$, metal chelators may prevent the occurrence of Fenton-type chemistry. Catalase (CAT) similarly impairs initiation by removing hydrogen peroxide, which is decomposed into non-radical species (eq. 2).

$$
2 \mathrm{H}_{2} \mathrm{O}_{2} \stackrel{\text { CAT }}{\longrightarrow} 2 \mathrm{H}_{2} \mathrm{O}+\mathrm{O}_{2}
$$

Chain-breaking antioxidants (also called radical-trapping antioxidants, RTA [3]) slow-down (or block) autoxidation by competing with the propagation reactions; i.e. they react with peroxyl radicals more rapidly than they can attack the oxidizable substrate, and, very important, their products of reaction do not propagate the autoxidation chain [2].

The most important and effective small-molecule-antioxidants are chain-breaking and phenols are the prototypical examples [3]: they are able to trap 2 peroxyl radicals per molecule of antioxidant, therefore they have a stoichiometric factor of $n=2$. Polyphenols, like dietary flavonoids, might instead trap more than 2 peroxyl radicals [25].

Their efficacy, however, primarily relates to the rate of reaction with peroxyl radicals (the actual chain-carrying species), which has to be compared to the rate of propagation, that is, the rate of $\mathrm{RH}$ + ROO•. The fact that a compound can react with "some radical species" does not mean it is an antioxidant, unless (i) the radical species is a peroxyl radical; (ii) the reaction is much faster than oxidative chain propagation; and (iii) the reaction products are species unable to propagate the chain-reaction [2-5]. Many dietary antioxidants, like isothiocyanates from brassica vegetables [8], are not provided of relevant antioxidant behavior, for example in the protection of linoleic acid in model systems; nonetheless they increase the antioxidant defenses in living systems, for example by inducing the expression of antioxidant enzymes (like glutathione reductase [EC 1.8.1.7] and glutathione peroxidase [EC 1.11.1.9]). They can be classified as indirect antioxidants as they only act by increasing the true (endogenous) antioxidants [2]. Conversely, compounds able to increase the overall rate of autoxidation in a system or able to increase the oxidative damage in a living cell, e.g. by depleting antioxidants or increasing the rate of radical generation, are called pro-oxidants $[2,6]$. The distinction between antioxidants and pro-oxidants should not be seen as a dichotomy, 
since it often depends on the system conditions and not infrequently known antioxidants in a model chemical system can act as pro-oxidants in living cells or a different model system [8,11,12,26,27]. One typical mechanism by which antioxidants can behave as pro-oxidants is the reduction of transition metal ions. By reducing, for instance, $\mathrm{Fe}^{3+}$ to $\mathrm{Fe}^{2+}$ or $\mathrm{Cu}^{2+}$ to $\mathrm{Cu}^{+}$, antioxidants might make these reduced forms available to reduce hydrogen peroxide or organic hydroperoxides in a Fenton-type reaction (see eq. 1), thereby increasing the rate of initiation [17]. Indeed, the mixture of iron salts and ascorbic acid is a well known pro-oxidant system (in the presence of $\mathrm{H}_{2} \mathrm{O}_{2}$ ) that turns into antioxidant at high concentration of ascorbic acid [28]. On the other hand, some antioxidants like flavonoids can simultaneously operate with the three different mechanisms, preventive, chainbreaking and indirect [11], with relative contributions that depend on experimental conditions. These aspects are actually a major source of controversy in antioxidant testing.

\section{Why focusing on peroxyl radicals?}

In the previous section we have stated that the quality of an antioxidant is related to its reactivity with peroxyl radicals. In doing so, not only we have neglected the "unnatural" persistent radicals commonly used in antioxidant assays (e.g. galvinoxyl, DPPH•, or ABTS•+, vide infra), whose relevance as models has already been questioned both to test antioxidant activity $[2,4]$ and to predict biological activity [12], we have also overlooked a variety of biologically relevant radicals.

For instance, gamma radiolysis of water (e.g. exposure of a living system to ionizing radiations) will produce the radical species summarized in eq. 3-6

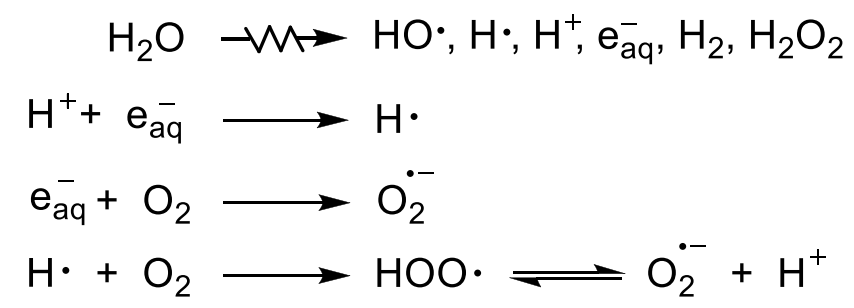

Solvated electron $\mathrm{e}_{\mathrm{aq}}{ }^{-}$is extremely reactive and will undergo diffusion controlled reaction both with protons (or oxonium $\mathrm{H}_{3} \mathrm{O}^{+}$) to yield hydrogen atoms (eq. 4), and with oxygen to yield superoxide 
radical anion (eq. 5) [29], which in turn will equilibrate with its neutral form $\mathrm{HOO} \bullet$ as depicted in eq. $6\left(\mathrm{p} K_{\mathrm{a}}=4.7\right)$ [30]. Hydrogen atoms are also extremely reactive with oxygen, to yield, again, superoxide (eq. 6). Hence the relevant radical species formed (in vivo as in vitro) in the early stages following gamma radiolysis are $\mathrm{H} \bullet, \mathrm{HO} \bullet, \mathrm{O}_{2}{ }^{-}$in equilibrium with $\mathrm{HOO} \bullet$. Why not testing the ability of antioxidants to quench them? Neutral superoxide $\mathrm{HOO} \bullet$ is not the prevailing species at physiological $\mathrm{pH}$, furthermore it has identical electronic structure and similar reactivity to peroxyl radicals [30]; therefore, knowing the reactivity of antioxidants with peroxyl radicals will also be indicative of their reactivity with $\mathrm{HOO} \bullet$ Conversely, superoxide anion has very modest reactivity with most antioxidants; its oxidation potential $\left(\mathrm{E}^{\circ}=-0.16 \mathrm{~V} v s \mathrm{HNE}\right.$ at $\left.\mathrm{pH} 7-14\right)$ indicates it is a reducing rather than an oxidizing species [30], and its role in biological systems is mainly as a chemical messenger that influences a multitude of redox-regulated processes [6,18,31]. Arguably, direct quenching of $\mathrm{O}_{2}{ }^{-\bullet}$ is not the prime role of exogenous antioxidants.

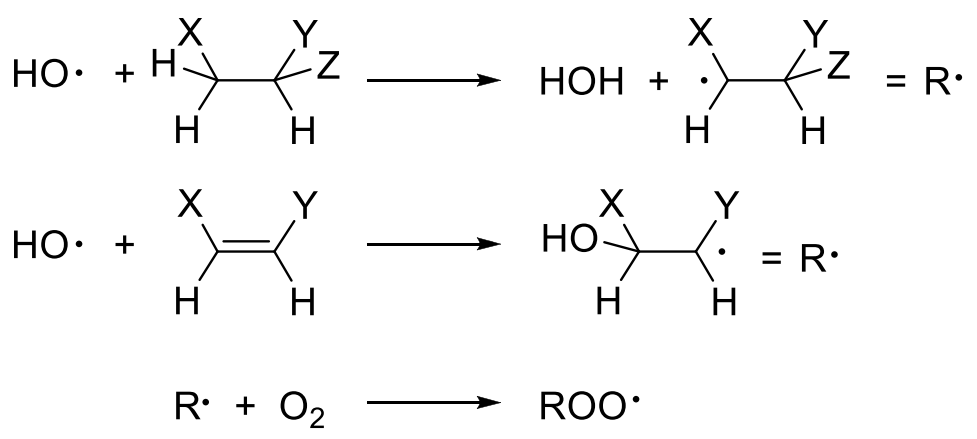

Hydroxyl radical and hydrogen atom have instead very high but unselective reactivity. This means that they react at close to diffusion controlled rate with most antioxidants; however, they react at similar rate also with most organic molecules. For instance the reaction of hydroxyl radicals with saturated hydrocarbons ( $\mathrm{C}-\mathrm{H}$ hydrogen abstractions, eq. 7) has been reported to occur with rate constants in the range $10^{8}-10^{9} \mathrm{M}^{-1} \mathrm{~s}^{-1}$ in water, being $\sim 2 \times 10^{9} \mathrm{M}^{-1} \mathrm{~s}^{-1}$ with saturated fatty acids [32], while addition to $\mathrm{C}=\mathrm{C}$ double bonds (eq. 8) has rate constants in the range $10^{9}-10^{10} \mathrm{M}^{-1} \mathrm{~s}^{-1}[33]$. Similar rate constants have been reported for the corresponding reactions of hydrogen atom in organic solution [34] and in water [35]. 
The lack of selectivity of $\mathrm{HO} \bullet$ and $\mathrm{H} \bullet$ indicates that it is impossible for an antioxidant to afford significant protection of an organic material, be it rubber or biomolecules, from these radical species under real-life conditions. It would require that the antioxidant and the material to protect are mixed in close to $1: 1$ ratio - a "dose" that would be unacceptable for any technological application and would kill any living system.

In all cases these reactions yield a carbon centered radical $\mathrm{R} \bullet$ that will react with oxygen at diffusion controlled rate to form a peroxyl radical (eq. 9). Peroxyl radicals would then propagate the damage by carrying an autoxidation chain-reaction (see scheme 1). The only protection that can be expected from an antioxidant comes from quenching those peroxyl radicals, thereby blocking the propagation.

\section{Chemical testing methods for antioxidants}

\section{Methods based on inhibited autoxidation}

These methods test antioxidants in close-to-real settings, i.e. they challenge their ability to inhibit the oxidation of a substrate. They are based on the comparison between the extent of the autoxidation of the substrate in the presence and in the absence of antioxidants. When they are performed with a constant rate of initiation (controlled conditions) and results are analyzed with a proper kinetic treatment, these methods are the most reliable to assess antioxidant activities.

The oxidizable substrate may be represented by purified natural compounds (e.g. linoleic acid and its esters, phosphatidylcholine, 7-dehydrocholesterol), by pure synthetic chemicals (styrene or cumene) or by natural lipid mixtures, such as egg yolk or lard, which however pose repeatability problems because of the variability in the composition and the presence of endogenous antioxidants $[15]$.

The initiation of the autoxidation can be spontaneous or induced. Spontaneous initiation is uncontrolled because it depends on traces of hydroperoxides in the starting material and on the exposure to light and/or heat. The addition of initiators reduces the reaction time and improves the 
reproducibility. Typical initiators are iron or copper ions which are able to decompose by dissociative electron transfer hydroperoxides, already present in the oxidizable substrates, or added on purpose (typically $\mathrm{H}_{2} \mathrm{O}_{2}$ ). This kind of initiation is not recommended because it suffers from autocatalysis due to the decomposition of hydroperoxides formed during the autoxidation, and from the direct interaction between the antioxidant and the metallic ions as previously discussed [15]. Azo-initiators, such as the lipid soluble AIBN (2,2'-azobis-isobutyronitrile), or water-soluble AAPH ((2,2'-azobis(2-amidinopropane) dihydrochloride) are much more useful, because their homolytic decomposition proceeds at a constant rate at a given temperature, during the entire course of autoxidation, providing a constant rate of initiation $\left(R_{\mathrm{i}}\right)$. The main difference among methods based on inhibited autoxidation is the way used to monitor the reaction.

A first group of methods is based on the measurement of oxygen consumption or of formation of hydroperoxides, the early products of autoxidation. They allow measurement of the absolute rate constants and of the stoichiometry of reaction between peroxyl radicals and antioxidants, hence they are best suited to gain detailed information about the mechanisms underlying the antioxidant action. Oximetry methods rely on the determination of $\mathrm{O}_{2}$ uptake in a closed system by using either a pressure gauge [36-37] (see figure 1) or a polarographic probe [38]. The main shortcoming of these techniques is their low throughput and the requirement of specialized instrumentation. A possible solution to these limitations has been proposed by Huang and co-workers, who showed that the autoxidation of methyl linoleate can be followed in a commercial 96-well microplate coated with an oxygen-sensitive fluorescence probe [39].

\section{<figure 1 here>}

In these experiments, the trend of the $\mathrm{O}_{2}$ consumption or hydroperoxides formation follows a typical biphasic behaviour: a first period in which the autoxidation is inhibited by the antioxidant (the induction period), followed by a fast rate of autoxidation, as shown in Figure 1. If the value of $R_{\mathrm{i}}$ is known, it can be used to obtain meaningful parameters describing the antioxidant action: the length of the inhibition period $(\tau)$ affords the stoichiometry of the tested antioxidant, while the 
comparison of the inhibited ( $R_{\mathrm{AH} 1}$ or $\left.R_{\mathrm{AH} 2}\right)$ and uninhibited $\left(R_{0}\right)$ oxygen-uptake rates afford the rate constant for the reaction of antioxidants with peroxyl radicals $k_{\text {inh }}$ (see Figure 1 ). The equations 10 and 11 are used, respectively in the case of good (AH1) and weak (AH2) antioxidants [40]. In these equations, $k_{\mathrm{p}}, 2 k_{\mathrm{t}}$ are, respectively, the rate constants for propagation and termination of the chosen substrate $[2,40]$

$$
\begin{gathered}
R_{A H 1}=\frac{k_{p}[\text { substrate }] R_{i}}{n k_{i n h}[A H 1]}+R_{i} \\
\frac{R_{0}}{R_{A H 2}}-\frac{R_{A H 2}}{R_{0}}=\frac{n k_{i n h}[A H 2]}{\sqrt{2 k_{t} R_{i}}}
\end{gathered}
$$

<scheme 2 here>

A conceptually different approach to obtain inhibition rate constants (but not the stoichiometry) is based on "peroxyl radical clocks", which consists in the quantisation of the various isomeric hydroperoxides formed during the autoxidation of linoleate or other oxidizable substrates [41]. Upon addition of an antioxidant to the autoxidizing substrate, as exemplified in scheme $2(\mathrm{XH}=$ $\mathrm{AH})$, the relative ratio of conjugated $(\mathrm{E}, \mathrm{F})$ over non conjugated $(\mathrm{G})$ hydroperoxides depends on the rate constant for reaction of the antioxidant with peroxyl radical $\left(k_{\mathrm{H}}\right)$, which can be determined with good accuracy by LC-MS analysis of the reaction mixture [41]. This approach has been implemented also with simpler substrates, allowing analysis to be conveniently performed by GC [42]. Kinetic data obtained by this method are usually in excellent agreement with those obtained by oxygen uptake kinetics.

Hydroperoxides, early products of lipid oxidation, can be quantified at time intervals by the iodometric assay, which consists in treating the sample with iodide: the oxidation of $\mathrm{I}^{-}$to $\mathrm{I}_{2}$ by hydroperoxides is followed by titration of $\mathrm{I}_{2}$ with thiosulphate. This technique is not specific because any compound that reacts with $\mathrm{I}^{-}$or $\mathrm{I}_{2}$ can interfere with it [43]. An improved method to monitor the formation of hydroperoxides has recently been proposed: it uses a fluorescent 
triphenylphosphine-coumarin probe whose emission increases upon oxidation by hydroperoxides, allowing the measurement to be performed in a fluorimeter or a microplate reader [44].

In the case of the autoxidation of natural non-conjugated polyunsaturated lipids, the formation of the typical absorption band of conjugated hydroperoxides at $232 \mathrm{~nm}$ can be monitored by spectrophotometry [45]. Determination of conjugated hydroperoxides by HPLC-UV analysis is however preferred to exclude interferences by other absorbing species that may be formed during the autoxidation [46].

Methods belonging to the second group rely on the detection of advanced oxidation products and provide only semi-quantitative information about antioxidant activity. Their main advantage is the higher throughput so they can conveniently be used for screening purposes, where they should be preferred to indirect assays (see below). These methods, as usually implemented, are unsuited to gain mechanistic information as they don't allow distinguishing preventive antioxidants from chainbreaking, unless the autoxidation is initiated by azo-initiators under controlled conditions.

The TBARS (thiobarbituric acid reactive species) assay is a popular method used to quantify lipid peroxidation. It based on the reaction of 2-thiobarbituric acid (TBA) with malondialdehyde (MDA), which is one of the advanced oxidation products of unsaturated lipids (eq. 12). Under acidic conditions and at high temperatures $\left(\sim 100^{\circ} \mathrm{C}\right)$, TBA and MDA react forming a pink coloured 2:1 adduct with a strong absorption $(532 \mathrm{~nm})$ and fluorescence emission $(553 \mathrm{~nm})$.<smiles>[Y2][SH]=C1NC(=O)CC(=O)N1</smiles>

Commercial TBARS assay kits are also available. In a typical experiment, the lipid samples are first peroxidized for a variable time lapse by using an initiating system (see above), then the extent of peroxidation, in the presence and in the absence of antioxidants, is assessed after a fixed time [47]. This method cannot distinguish between the kinetics and the stoichiometry of reaction. It also suffers from limitations due to reactions of TBA with other compounds not related to lipid 
peroxidation, and to the formation of Schiff bases between malondialdehyde and amines, misestimating the antioxidant protection [43].

Secondary oxidation products other than MDA can been used to follow the autoxidation of unsaturated lipids: in particular volatile products such as hexanal, which can be analyzed by headspace gas chromatography [48] or 4-hydroxynonenal (4-HNE) that can be analyzed by GC-MS or HPLC after appropriate derivatization [49].

The Rancimat apparatus measures the release of volatile acids, formed upon the spontaneous oxidation of fats (such as seed oils or lard) under an air stream at $90-120^{\circ} \mathrm{C}$, by a conductometric method [50]. The antioxidant activity is described as a function of the induction time observed in the oxidation profiles and it is indicated as Oil Stability Index (OSI). The antioxidant effect is expressed as Protection Factor (PF), defined as the ratio between the OSI in the presence and that in the absence of antioxidant. This method provides an estimate of the antioxidant stoichiometry combined with threshold reactivity. The high temperature might cause the loss of low-boiling or labile antioxidants, resulting in underestimation of their activity.

\section{Methods Based on Competitive Probe Reaction.}

In these assays, antioxidants prevent the reaction between peroxyl radicals and an oxidizable probe, whose reaction can be easily detected by some spectroscopic technique (UV-vis, fluorescence, EPR). The most important reaction underlying these methods are summarized by equations 13-17, where $\mathrm{PH}$ (or $\mathrm{P}$ ) and $\mathrm{AH}$ are, respectively, the probe and the antioxidant. The result is dependent on the reactivity of the probe itself with peroxyl radicals $\left(k_{15}\right.$, by formal hydrogen abstraction or radical addition) and on the thermodynamics of equilibrium 17 [51]. 


$$
\begin{aligned}
\text { initiator } \stackrel{\mathrm{O}_{2}}{\longrightarrow} \mathrm{ROO} \cdot \\
2 \mathrm{ROO} \cdot \stackrel{\text { non radic. prod. }}{\longrightarrow} \mathrm{ROOH}+\mathrm{P} \cdot \\
\mathrm{ROO} \cdot+\mathrm{PH} \stackrel{k_{15}}{\longrightarrow} \mathrm{ROO} \\
\mathrm{ROO} \cdot+\mathrm{P} \stackrel{k_{15}}{\longrightarrow} \mathrm{ROO}-\mathrm{P} \cdot \\
\mathrm{ROO} \cdot+\mathrm{AH} \stackrel{k_{16}}{\longrightarrow} \mathrm{ROOH}+\mathrm{A} \cdot \\
\mathrm{P} \cdot \text { (or ROOP.) }+\mathrm{AH} \stackrel{K_{17}}{\longrightarrow} \mathrm{PH}(\text { or } \mathrm{ROOPH})+\mathrm{A} \cdot
\end{aligned}
$$

ORAC (oxygen-radical antioxidant capacity) is a popular method used to estimate the content of antioxidants in food. The antioxidant competes with a fluorescent probe for quenching peroxyl radicals generated from AAPH. The first probe that was proposed was a fluorescent protein, phycoerythrin [52], that was later replaced by the more practical fluorescein (Scheme 3), a phenolic derivative with a fluorescent emission at $520 \mathrm{~nm}$ on excitation at $480 \mathrm{~nm}$ [53]. This assay is suitable to be performed in a parallel fashion in thermostatted microplate readers for high throughput analysis of several samples, so that extensive databases of the antioxidant power of foods measured by the ORAC method are available, although the nutritional relevance of these data has recently been questioned [54]. The main limitation of ORAC assay is the use of the Area Under the Curve (AUC) approach to measure the protective effect of antioxidants (see next section for a discussion about AUC) and the low reactivity of fluorescein toward ROO • radicals, that even out the result for good antioxidants. An interesting improvement of the ORAC assay has been reported by LópezAlarcón and Lissi, who suggested the use of pyrogallol red (Scheme 3) as a probe for competitive antioxidant assays [55]. As pyrogallol red reacts faster than fluorescein with ROO• radicals, its consumption does not present induction times, even in the presence of very reactive antioxidants, with the exception of ascorbic acid. The authors suggested that pyrogallol red should be used as a complementary probe of fluorescein to estimate the antioxidant behaviour of complex mixtures. The $\boldsymbol{\beta}$-carotene bleaching test consists in measuring the decay of the absorption at $470 \mathrm{~nm}$ due to $\beta$-carotene under a flux of free radicals in the presence or absence of antioxidants. The reaction is 
performed in an aqueous emulsion of linoleic acid prepared using Tween ${ }^{\circledR} 40$ as phase stabilizer. Radicals are generated by the spontaneous oxidation of linoleic acid at the temperature of the test $\left(50{ }^{\circ} \mathrm{C}\right)$. The antioxidant activity is expressed as $\%$ inhibition with reference to the control after 60 min incubation [56]. The lack of reproducibility of initiation, the complexity of the reaction involving carotenes under $\mathrm{O}_{2}$ - they have antioxidant action at low $\mathrm{O}_{2}$ concentration, while they propagate the oxidative chain in air saturated solutions [57] - and the crude kinetic treatment are the main limits of this test.

Crocin bleaching test is based on the reaction between crocin (Scheme 3), a natural water soluble carotenoid contained in flowers of the Crocus genus, and peroxyl radicals generated by thermal decomposition of AAPH. Antioxidants are able to retard the decrease of the $443 \mathrm{~nm}$ absorbance of crocin caused by the attack of peroxyl radicals. The antioxidant activity is expressed as the ratio between the rates of crocin bleaching in the absence and in the presence of antioxidants; therefore, it provides the reactivity and not the stoichiometry of the antioxidant [58].

<scheme 3 here>

Spin-trapping methods. Peroxyl radicals generated by the decomposition of AAPH react with a spin-trap, such as CYPMPO [5-(2,2-dimethyl-1,3-propoxy cyclophosphoryl)-5-methyl-1-pyrroline$\mathrm{N}$-oxide], forming a relatively stable nitroxide that can be quantified by electron paramagnetic resonance (EPR) at a fixed time (eq. 18) [59]. The antioxidant reacts with peroxyl radicals faster than the spin-trap, thus inhibiting the formation of the nitroxide. These methods have no interference from the sample colour and turbidity, since EPR is selectively sensitive to unpaired electrons, but they don't distinguish between kinetics and stoichiometry, and may be influenced by the possible antioxidant action of the nitroxides [60]. These methods typically provide semiquantitative data, since most spin adducts have limited life-time and their decay in the time lapse between trapping and measurement needs to be taken into account to achieve really quantitative results. 


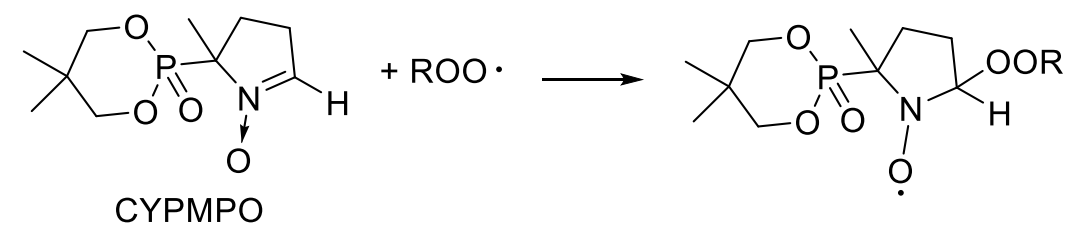

Luminol method. Peroxyl radicals generated by the decomposition of AAPH react with luminol $\left(\mathrm{LH}_{2}\right)$, to generate a luminol radical $(\mathrm{LH} \bullet)$ which is further oxidized in the presence of $\mathrm{O}_{2}($ scheme 4), with a mechanism not completely understood, to an endoperoxide with the loss of $\mathrm{N}_{2}$. Finally, the bond between oxygen atoms in the endoperoxide group breaks, generating aminophthalic acid and emission of blue light centred at $\sim 425 \mathrm{~nm}$ [61]. Luminescence is measured by a luminometer or a scintillation counter. When antioxidants are present, luminescence is inhibited until the antioxidants are exhausted, and the time of inhibition is proportional to the total concentration of antioxidants. Thus this technique affords a measure of the stoichiometry of the antioxidants or their concentration in a matrix of unknown composition. They provide no indication of their reactivity or efficacy. It is also unclear if the lag time is due to the scavenging of peroxyl radicals by antioxidants, or it is due to the back reduction of $\mathrm{LH} \bullet$ radicals [62].

<scheme 4 here $>$

Total oxidant scavenging capacity (TOSC) assay is based on oxidation of alpha-keto-gamma(methylthio)butyric acid (KMBA) to ethylene by peroxyl radicals produced from AAPH. The antioxidant capacity of a molecule is quantified from its ability to inhibit ethylene formation by measuring the area under the curve (AUC) of ethylene concentration versus time. The reaction is followed by headspace gas-chromatography (HS-GC), so it doesn't suffer the interference of coloured matrices [63]. The main limitation of this technique is the long reaction time and the necessity of multiple chromatographic analyses for each experiment. The use of AUC approach also reduces the chemical significance of results. 


\section{Indirect Methods.}

In these methods, coloured persistent radicals are used as probes. They are reduced by the antioxidant, and the colour change in the solution is measured by spectrophotometric techniques [58]. The seemingly convenience of these methods is obscured by their major limitations, which make them suitable only for preliminary screening procedures. The probes are chemically very different from the radicals responsible for the autoxidation of real systems, so the scavenging reaction doesn't often follow the same mechanism as observed with peroxyl radicals [15]. Due to their experimental design, these assays make it impossible to test if the investigated compound is really capable of interrupting the chain reaction of autoxidation, or if it is simply able to reduce the probe, as we and others have previously discussed in detail in the case of very oxidizable essential oils $[4,64]$. These stable radicals may react with reductants having no antioxidant activity, for instance DPPH• is completely reduced to DPPHH also by $\mathrm{H}_{2} \mathrm{O}_{2}$, that can hardly be considered an antioxidant [65]. Therefore, the results obtained with these methods indicate a "radical trapping power" or a "reducing power" rather than true antioxidant activity. Single-point measurements of absorbance decay after a fixed time (which usually arbitrarily varies from laboratory to laboratory) further reduces the meaning of these methods (see the following section).

DPPH Test. An antioxidant, or any molecule with a weak $\mathrm{X}-\mathrm{H}$ bond, reacts with the coloured and highly persistent radical DPPH• (2,2-diphenyl-1-picrylhydrazyl, $\lambda_{\max } \approx 520 \mathrm{~nm}$ ) causing discoloration of the solution (eq. 19) [50,55].
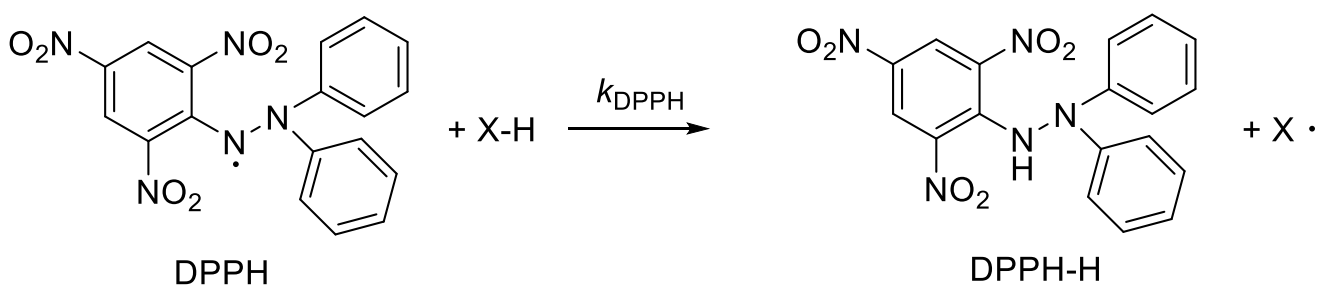

Results are commonly expressed as $\mathrm{IC}_{50}\left(\right.$ or $\left.\mathrm{EC}_{50}\right)$, defined as the concentration of the potential antioxidant needed to decrease by $50 \%$ the initial absorbance of the coloured radical. Because it depends on the reaction time, taken alone this parameter does not provide meaningful information 
of the actual reactivity of the antioxidant; furthermore, data can only be compared when obtained under identical settings. The significance of this method could be greatly improved by monitoring the entire time evolution of the reaction instead of performing single-point measurements. Due to the similar electronic configuration between DPPH• and peroxyl radicals, the rate constants for the reaction between the two radicals and phenolic antioxidants, although different by about three orders of magnitude (see Table 1), have the same ranking [66]. The bimolecular rate constant for the reaction between DPPH• and various phenols, that is usually measured by using an excess of antioxidant under pseudo-first order conditions, allows to clarify the structure-activity relationship of families of antioxidants, such as flavonoids [67], and their reaction mechanisms [68]. However, the linear relationship between the rate constants with $\mathrm{DPPH} \bullet$ and ROO• only holds in aprotic solvents, whereas in polar protic solvents (i.e. ethanol or methanol: DPPH• is insoluble in water) the reaction between DPPH• and phenols is strongly accelerated by a stepwise proton-transfer electron-transfer mechanism (named SPLET) [69] (see Scheme 5).

<scheme 5 here>

Litwinienko and Ingold have shown that, when using $\mathrm{MeOH}$ as solvent, acidic phenols react much faster than expected with DPPH• [69], which would subvert the order of reactivity with respect to their actual antioxidant activity. In Table 1 are collected some results that they obtained in the case of the family of phenols, compared to their reactivity with peroxyl radicals [70].

<table 1 here>

It is unfortunate that ethanol or methanol are the most typical solvents used to perform the DPPH assay, particularly when testing natural extracts: acetonitrile or dioxane would act equally well in solubilising modest concentrations of polar antioxidants and would yield results unbiased by the SPLET mechanism. 
The TEAC Test (Trolox-Equivalent Antioxidant Capacity) is based on the reaction with the colored and relatively persistent 2,2'-azinobis(3-ethylbenzothiazoline-6-sulfonic acid) (ABTS ${ }^{+\bullet}$ radical cation (or monoanion, if the two sulfonate groups are considered), which has a strong absorption band at $734 \mathrm{~nm}\left(\varepsilon_{734}=0.015 \mu \mathrm{M}^{-1} \mathrm{~cm}^{-1}\right)$ (eq. 20).<smiles>[Y20]S(=O)(=O)c1ccc2c(c1)s/c(=N\N=c1/sc3cc(S(=O)(=O)OCC)ccc3n1CC)n2CC</smiles>

The radical is generated in buffered water just before the test by reacting ABTS with an oxidizer, such as potassium persulfate or $\mathrm{MnO}_{2}$. The antioxidant activity is defined as the amount of $\mathrm{ABTS}^{+\bullet}$ quenched after a fixed time (usually $5 \mathrm{~min}$ ) and is compared with that produced by Trolox ${ }^{\circledR}$ [71]. If performed in this way, this test does not distinguish between kinetics of radical trapping and stoichiometry, and the outcome may depend on the time chosen before reading the absorbance [72]. By following the time course of the reaction for longer periods, Perez-Jimenez and Saura-Calixto have shown that $\alpha$-tocopherol and ascorbic acid have a stoichiometry slightly larger than $n=2$, that is the number expected from the "usual" radical trapping mechanism of phenolic antioxidants, while other inhibitors have significantly larger values (resveratrol: $n=6$; quercetin: $n=7$ ). On the other hand, the time required to reach the stationary concentration, which inversely depends on the rate constant for the reaction between antioxidants and $\mathrm{ABTS}^{+\bullet}$, widely varies among antioxidants, being smaller for $\alpha$-tocopherol and ascorbic acid [73]. An important limitation of this assay is that $\mathrm{ABTS}^{+\bullet}$ is a radical cation while the peroxyl radical is neutral, so antioxidants react with $\mathrm{ABTS}^{+\bullet}$ by an electron transfer mechanism, whereas with peroxyl radicals they react by formal $\mathrm{H}$-atom transfer [66].

Galvinoxyl test. Galvinoxyl is a stable phenoxyl radical having a strong absorption peak at $428 \mathrm{~nm}$ in ethanol. In the presence of phenolic compounds or other antioxidants, it is reduced by electron (and proton) or hydrogen-atom transfer to the colourless phenol (eq. 21) [74]. Its use to assess 
antioxidant activity has similar advantages and limitation of the other indirect methods, previously described.

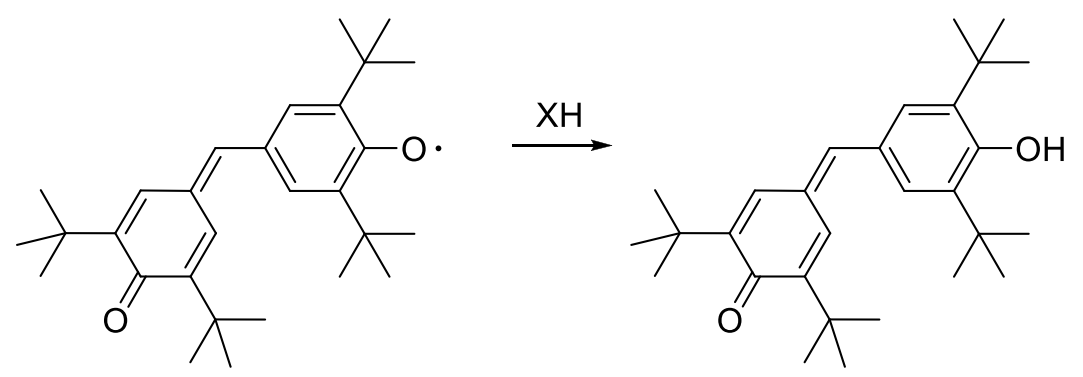

Indirect methods not involving ( $s-p)$ radical chemistry.

These methods are based on the reaction of potential antioxidants with inorganic oxidants. Although some of such inorganic species might be paramagnetic $\left(\right.$ e.g. $\mathrm{Fe}^{3+}$ or $\left.\mathrm{Cu}^{2+}\right)$, their unpaired electrons (if present) reside in d-f orbitals and their reactivity is not representative of that of organic radicals like peroxyls (with the unpaired electron in s-p orbitals). These methods test the reducing ability not the antioxidant activity.

FRAP Test (Ferric Reducing Antioxidant Power). The antioxidant reduces $\mathrm{Fe}^{3+}$ to $\mathrm{Fe}^{2+}$, which forms a coloured complex $(593 \mathrm{~nm})$ with 2,4,6-tripyridyl-s-triazine (Fe $\left.\mathrm{Fe}^{\mathrm{II}} \mathrm{TPTZ}\right)$ in acetate buffer, pH 3.6. The amount of Fe $\mathrm{F}^{\mathrm{II}}$-TPTZ produced is measured spectrophotometrically after a fixed time (4 $\min$ ) [75] Alternatively, iron reduction can be assessed by the potassium ferricyanide-ferric chloride method [50]. This method essentially provides the stoichiometry of antioxidants, which for instance has been determined as two for ascorbic acid, uric acid and $\alpha$-tocopherol, about 4 for bilirubin and zero for albumin. The reaction with ascorbic acid is instantaneous, whereas with uric acid it takes about two minutes to occur, so after 4 min both antioxidants have the same FRAP power. However, in the case of other antioxidants the reaction is not complete after 4 minutes, so the result of this test is expected to arbitrarily depend on the reaction time. The reaction is nonspecific, and any compound with a suitable redox potential will drive Fe $\mathrm{F}^{\mathrm{III}}-\mathrm{TPTZ}$ reduction [75] The CUPRAC test (Cupric Reducing Antioxidant Capacity) is conceptually similar to the FRAP 
test, but is based on the reduction of $\mathrm{Cu}^{2+}$ ions in the presence of neocuproine (2,9-dimethyl-1,10phenanthroline) at $\mathrm{pH} 7$, which involves faster kinetics [76].

Folin-Ciocalteu Test. The test was originally designed to titrate polyphenols in wine, but it has then been arbitrarily extended as a method to measure phenols or antioxidants in food or vegetable extracts. Phenols are oxidized in a basic medium by a mixture of tungstate and molybdate (Folin-Ciocalteu reagent) with the consequent formation of coloured molybdenum ions, $\mathrm{MoO}^{4+}$ $(750 \mathrm{~nm})[50]$. Although this test is claimed to provide the total phenolic content of a sample, it is not specific for phenols, or in general for antioxidants: any reducing agent will get good score with this test and be assigned as an antioxidant. Results are reported relative to gallic acid, although other phenols have also been used.

Nanoparticle-Based Assays of Antioxidant Activity. These methods are based on the peculiar properties of metal nanoparticles: large superficial area, which facilitates surface redox reactions, and the presence of plasmonic bands that confer high absorbance in the visible. Despite their conceptually innovative chemistry, their limitations are the same as the other indirect methods not involving radical chemistry. A test based on gold nanoparticles has been proposed by Scampicchio et al. Antioxidants reduce $\mathrm{Au}^{\mathrm{III}}$ ions to $\mathrm{Au}^{0}$, thus causing the formation of coloured nanoparticles in the presence of citrate and a surfactant. The formation of nanoparticles is assessed by spectrophotometric readings at $555 \mathrm{~nm}$. The results of this test are reported to be similar to the Folin-Ciocalteu test [77]. Another example is that of cerium oxide $\left(\mathrm{CeO}_{2}\right)$ nanoparticles used for developing a portable colorimetric assay for antioxidants. The test is based on the reduction of $\mathrm{Ce}^{\mathrm{IV}}$ into $\mathrm{Ce}$ III on the surface of the nanoparticles, with the development of a brownish colour. $\mathrm{CeO}_{2}$ nanoparticles can be immobilized on filter paper, to obtain an easy to use sensor.

Also in the case of these tests, a reducing activity rather than antioxidant activity is determined [78].

\section{The importance of kinetic data treatment}

In the previous section we have discussed the chemistry behind some typical assays of antioxidant 
activity. An important aspect that has major influence on the significance of results is the way data, obtained by those assays, are treated and presented. We will illustrate the concept taking the very popular ORAC assay as example.

\section{$<$ figure 2 here>}

Figure 2 shows simulated ORAC plots for three antioxidants differing for reactivity with peroxyl radicals $\left.\left(k_{\mathrm{ROO}}\right)^{\circ}\right)$ and stoichiometry of reaction $(n)$ as indicated in the insert. Each plot illustrates the ability of the corresponding antioxidant in sparing fluorescein from attack by peroxyl radicals (with loss of fluorescence), by reacting faster than fluorescein with peroxyl radicals. In other words, in ORAC assay fluorescein represents the "biomolecule" to protect. From the plots it is clear that antioxidant AH1 is the most effective, being able to completely spare fluorescence until it is consumed. By comparison, antioxidant $\mathrm{AH} 2$, being 2-fold slower in trapping peroxyl radical and having the same stoichiometry of reaction $(n=2)$, does not give neat inhibition and allows loss of fluorescence at any time, albeit at reduced rate. Analysis of those data with the standard method of the area under the curve (AUC) of each plot (minus the AUC of the reference plot without antioxidant to yield $\triangle \mathrm{AUC}$ ), indicates that antioxidant AH1 is only marginally more effective than $\mathrm{AH} 2$, (15 vs 11 a.u.), while kinetic analysis of those plots would reveal it is actually twice as effective. Comparison with antioxidant AH3 is even more striking. This compound is 5-fold less effective than AH1 in trapping peroxyl radicals but has double stoichiometric factor, meaning that its action will last longer. Graphical inspection of the corresponding ORAC plot indicates that AH3 gives much lower protection than AH1 being only able to slow down the decay of fluorescein. This will correspond to similar differences in real-life antioxidant performance. However, $\triangle \mathrm{AUC}$ values indicate that $\mathrm{AH} 3$ is a better antioxidant than $\mathrm{AH} 1$. Therefore, while kinetic analysis would allow distinguishing the stoichiometry of reaction (the duration of protection) from the rate constant of peroxyl radical trapping (the efficacy of protection), the method of AUC mixes the two parameters, 
giving more weight to stoichiometry than to reactivity and yielding numerical values of no physical meaning, which might be completely misleading in judging the antioxidant performance. This represents the main limit of the ORAC assay, and its significance could be largely improved by performing full kinetic data analysis.

As discussed in the previous section, the very popular DPPH assay is based on the quenching by the antioxidant of violet and persistent $\mathrm{DPPH} \bullet$ radical, monitoring the reaction by spectrophotometry. Figure 3 displays the simulated time-course of the reaction for three antioxidants differing for reactivity $\left(k_{\mathrm{DPPH}}\right)$ and stoichiometry of reaction $(n)$ with DPPH $\bullet$ In the example, the order of efficacy of antioxidants in quenching DPPH $\bullet$ is: $\mathrm{AH} 1>\mathrm{AH} 2>\mathrm{AH} 3$, reflecting the corresponding rate constants, which is immediately obvious from visual inspection of the plots. However, it is common practice in DPPH assay to express results as IC50 or as \% discoloration of the solution after a fixed time. The two forms are equivalent and are referred to a single measurement at fixed time. Typical reaction time is 30 minutes, however it may vary from lab to lab. Reaction of faster antioxidant $\mathrm{AH} 1$ is completed after about 12 minutes, antioxidant $\mathrm{AH} 2$ takes about 30 minutes to completion while reaction of $\mathrm{AH} 3$ is still continuing after 60 minutes. In the example, if the \% discoloration is determined at 30 minutes the three antioxidants appear having identical performance, despite the substantial difference in reactivity. Instead, measurement at 10 minutes would give the correct order of reactivity $(\mathrm{AH} 1>\mathrm{AH} 2>\mathrm{AH} 3)$, although numerical values are not in scale with the actual rate constants. It should be noted that a measurement taken at 60 minutes would yield a completely different order of performance, $\mathrm{AH} 3>\mathrm{AH} 1=\mathrm{AH} 2$, merely reflecting the stoichiometry of reaction.

\section{<figure 3 here>}

As a consequence, assays based on single-point measurements after long reaction time should be regarded as titrations of the reducing components in an extract, for instance, but provide no information on the antioxidant activity of the test compound(s). Assays based on short reaction time 
(1-10 minutes, depending on the type and concentration of the reactants) are more likely to yield at least the correct ranking of efficacy among different antioxidants. Since recording the full time course of the reaction (as in figure 3) is straightforward with today equipment, it would be equally convenient and much more valuable to perform full kinetic analysis of DPPH• quenching, obtaining actual rate constants as a significant indicator of the reactivity with this model species $[45,67]$. The DPPH• example explains, at least in part, the large variability encountered from study to study on measurements performed in a single-point fashion. Indeed the same considerations also apply, for instance, to TEAC test and all indirect tests, TBARS or secondary products measured in autoxidizing mixtures, $\beta$-carotene and crocine bleaching tests, the luminol method, etc. In all such cases, results depend on the chosen reaction time and conditions, as well as on the stoichiometry of reaction and concentration of the test compound, beside its reactivity, often yielding inconsistent results.

\section{Cell-based assays and in vivo testing of antioxidants}

Appropriate chemical assays can provide valuable data on the performance of antioxidants in protecting molecules from oxidative damage. However, when antioxidants are investigated as potential drugs or bioactive food factors, a number of additional parameters become relevant in determining their efficacy in a living system. First of all, their "bioavailability", meant, in a broad sense, as their ability to localize at useful concentration where they are needed. Compartmentation of biological systems is a main issue to consider: even excellent water-soluble antioxidants like vitamin $\mathrm{C}$ will perform poorly in protecting from radical damage localized in the core of a phospholipids bilayer (e.g. a cell membrane) [79]. On increasing the complexity of the biological system, factors like absorption by the selected way of administration, distribution and metabolism will become more and more relevant. These aspects cannot be accounted for by chemical assays. Furthermore, chemical assays cannot measure indirect antioxidant activity, which is only expressed in a living system. While in vivo testing implies high costs and may raise ethical issues, a wealth of 
cell-based assays have been described as surrogates or preliminary screening. They are typically based on: (a) selecting a representative cell line, (b) culturing it under standardized conditions, (c) subjecting the cell culture to some source of oxidative stress in the presence or absence of the antioxidant, (d) assessing oxidative damage in treated versus untreated cells. Each of those steps represents a critical point that will influence the results, and differences among the many assays described in the literature mainly consist in different choices concerning such points.

Selecting representative cells is arguably a major issue that might compromise the significance of results $[19,80]$. Primary cells isolated from animal or human tissues might have close to in vivo metabolism; however they live shortly in culture and are often supplied by different donors, causing low reproducibility of results [19]; they also easily change gene expression and metabolism, becoming less representative than expected [81]. Immortalized cell lines are easier to handle and of broader use: a variety of models have been proposed for specific investigations [21]. Since liver has a major role in lipid metabolism, human liver HepG2 cell line has been proposed as golden reference for antioxidant testing [82]; however this choice has been criticized, based on the finding that HepG2 cells have increased catalase expression and altered response to oxidative stress [83]. Among other proposed models, human colon HT29 and Caco-2 lines are often encountered in the literature [19]. Different results can be found by testing the same antioxidants in different cell-lines, and human erythrocytes have been suggested as representative biological model $[83,84]$. Beside the chosen cell line, the culture medium with its influence on cell metabolism plays a very critical role. Cell culturing itself may induce oxidative stress, for instance due to the non-physiological absence of some antioxidants in the culture medium [19]. This may exaggerate the benefits of antioxidant treatment or, conversely, may induce adaptation mechanisms, thereby altering cell response. Decreased sensitivity to oxidative stressors could occur, for instance, by induced production or release of metabolites like pyruvate or oxaloacetate, both increasing resistance to $\mathrm{H}_{2} \mathrm{O}_{2}$ [85]. Interactions of the culture medium with the antioxidant, such as precipitation, complexation or chemical reaction should also be taken into account. Another frequently overlooked criticism could 
come from the artificial induction of oxidative stress: oxidative insult is often massive and not representative of physiological conditions. It is obtained, for instance, by addition in the culture medium of variable amounts of AAPH, hydrogen peroxide, tert-butyl hydroperoxide or other stressors. Under "extreme" conditions the protection offered by antioxidants may not be representative of their performance in vivo. Recently, dietary fatty acids have been proposed as more physiological stressors [86]. Finally, a very important point that varies among different assays is the method chosen to assess oxidative damage. The most frequent end-point is analysis of cell survival in the presence/absence of the antioxidant, commonly performed via the MTT assay [87]. Although this is clearly a relevant end-point, it implies rather extreme test conditions, as the study needs to be set-up so that modest cell survival is registered in untreated cells. As previously discussed, this may not be representative of physiological settings.

Furthermore, decreased or increased cell viability could be unrelated to the actual antioxidant activity and depend on different pharmacological activity [88]. This is particularly the case when vegetable extracts of uncertain or complex composition are tested as potential antioxidants. Other popular assays are not free of fault. The use of 2',7'-dichlorofluorescein (DCF) to measure oxidative stress in cells is very popular $[18-20,86]$ and convenient since it does not require sophisticated equipment or analytical skills. It is based on administration to the cell culture of the reduced diacetate form, DCFH-DA, which is hydrolyzed to DCFH inside the cell and yields intense fluorescence upon oxidation to DCF. Therefore the intensity of fluorescence is taken as a measure of radical production or the oxidative stress inside the cell. Unfortunately, it has been shown by EPR studies that DCFH itself is able to generate radicals and oxidative stress inside the cell, under aerobic conditions, in a visible light-dependent process [89]. As a consequence, using the DCF assay to probe or measure oxidative stress in biological systems has been judged a "self-fulfilling prophesy", exposing results to serious artifacts [90]. A recent better approach to measure the oxidative status of cells is based on a novel fluorescent probe built by connecting the $\alpha$-tocopherol core to a BODIPY fluorophore, whose fluorescence is activated when the tocopherol moiety traps a 
peroxyl radical [91]. An alternative approach is based on the radical-probe technique: cells are shortly incubated with an hydroxylamine probe, which is readily oxidized to the spin-active persistent nitroxide inside the cells by most physiological oxidizing species, and the nitroxide can be reduced back, still inside the cell, by physiological reducing systems as illustrated in eq. 22 [92].

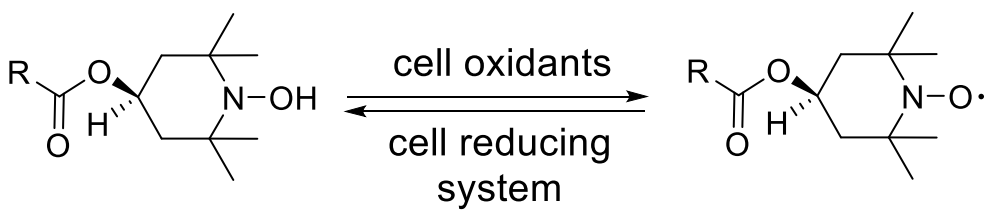

Therefore, measuring the equilibrium concentration of nitroxide will provide a quantitative estimate of the red-ox balance inside the cell [92]. One advantage of this method is that it can be applied also in animal or human tissues ex-vivo [93-95], as well as in whole animals [14], following in vivo treatment with antioxidants. Other testing methods include the analytical determination of specific post-facto markers of oxidative damage, such as isoprostanes, aldehydes (e.g. MDA, malondialdehyde), DNA damage markers (e.g. 8OHdG, 8-hydroxy-2'-deoxyguanosine): their advantages and limitations have been discussed in detail [18]. None of them, taken individually, appears to represent a "best" reference marker of oxidative damage; however, determination of a selection of them can provide a representative picture. All these markers can also be determined in animal tissues ex-vivo following in vivo treatments [18]. Another interesting approach consists in measuring the activity/expression of antioxidant or pro-oxidant enzymes following antioxidant treatment in cell culture or in vivo $[96,97]$. These studies may not offer a global picture of the antioxidant performance but bring very relevant mechanistic information [98] that is difficult to obtain by other assays: they allow measuring the indirect antioxidant (or pro-oxidant) behavior of test molecules [8]. Measuring multiple markers of oxidative damage as well as the activity of antioxidant enzymes offers a valuable overview of the biological potential of antioxidants, although the analytical work behind such kind of investigation could be highly time-consuming and not suited to the rapid screening of many samples. 


\section{Testing of preventive antioxidant activity}

Preventive antioxidant activity is most commonly expressed by inhibition of the Fenton-type chemistry, which might be involved in the initiation process. On a mechanistic perspective this could be due to chelation of transition metal ions and/or removal of peroxides, hydroperoxides or hydrogen peroxide. Inhibited autoxidation studies could be useful also in testing preventive antioxidants, in case initiation is obtained by Fenton chemistry (i.e. addition of $\mathrm{H}_{2} \mathrm{O}_{2}$ and $\mathrm{Fe}^{2+}$ or $\mathrm{Cu}^{+}$to the oxidizable mixture), by comparing the results with those obtained in identical autoxidations initiated by azo-compounds (whose decomposition is normally not affected by antioxidants), so to distinguish chain-breaking from preventive antioxidant behaviour [99]. Additionally, metal-binding studies could serve to quantify the ability of the antioxidant in chelating transition metal ions [100]. Removal of hydroperoxides and hydrogen peroxide could be achieved in a stoichiometric reaction, as illustrated in eq. 23 for glucoerucine being converted in glucoraphanine. The reaction kinetics can conveniently be monitored by mass spectrometry [101].<smiles>CS/C(=N\O[Na])C(C)SC</smiles><smiles>CS(=O)CC(=NO[Se])SC1CCCCC1</smiles>

Other preventive antioxidants, however, remove peroxides in a catalytic fashion, i.e. they catalyze the dismutation of hydrogen peroxide, thereby mimicking CAT, or the reduction of peroxides at the expenses of sacrificial reductants, thereby mimicking peroxidases like glutathione peroxidase (GPx; [EC 1.11.1.9]).

CAT-mimetic activity. Hydrogen peroxide has a broad unresolved absorption band in the UV region up to $400 \mathrm{~nm}$, hence its kinetics of disappearance in the presence of a CAT-mimic, prompting its decomposition to water and molecular oxygen (eq 2), can be monitored directly by spectrophotometry in the range $200-300 \mathrm{~nm}$ (tipycally $240 \mathrm{~nm}$ ), as illustrated in the original work of Beers and Sizer, which, together with its modifications, is the best established method in the literature [102]. 
The method has recently been implemented for use in a 96-dwell microplate reader to increase the throughput [103].

Commercial assay kits, offering improved sensitivity with respect to the classical spectrophotometric method, are based on the reaction of unconverted hydrogen peroxide with a probe such as N-acetyl-3,7-dihydroxyphenoxazine (Amplex Red) in the presence of horseradish peroxidase (HRP; EC 1.11.1.7) to form a coloured and fluorescent product, resorufin, that can be quantified either by spectrophotometry or by fluorimetry [104]. The method is sensitive and can be applied directly in microplate readers; however, when testing synthetic CAT-mimics, care should be taken to avoid artifacts related to interaction of the test molecule with HRP or with the probe. For instance, it has been reported that production of resorufin by the Amplex Red/HRP system is promoted by NADH and reduced glutathione in biological systems, leading to aberrant signals [105].

Since the spectrophotometric measurement of hydrogen peroxide can be biased by other species having absorbance in the same region, polarographic detection of oxygen formation by a Clark-type electrode has been proposed as alternative, which can be implemented both in aqueous [106] or apolar media [107].

On monitoring oxygen release, however, the linearity might be limited for high CAT activity by the evolution of oxygen from the solution. This limit has actually been exploited to design a recent very simple method that provides quantitative CAT activity data with minimal equipment. It is based on monitoring the height of foam formation when oxygen bubbles are formed in the presence of the neutral surfactant Triton X-100, upon calibration with standard CAT solutions [108].

The authors claim lower sensitivity but similar linearity and precision than conventional spectrophotometric methods [102].

GPx-mimetic activity. One of the most efficient way to remove hydrogen peroxide in nature is the catalytic cycle of glutathione peroxidase, a family of Se-containing enzymes which convert $\mathrm{H}_{2} \mathrm{O}_{2}$ in water (eq. 24) using glutathione (GSH), a thiol, as stoichiometric reducing agent. 


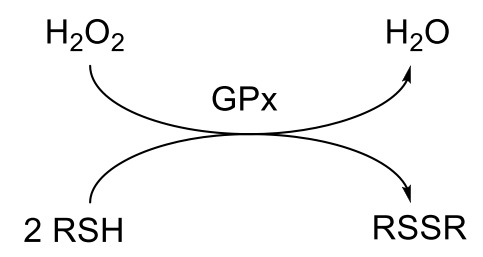

The major interest in the search of GPx-mimic antioxidants has stimulated the development of several assays, which have been the subject of a specific review [109]. The most typical assay is indirect, requiring the GPx-mimic, hydrogen peroxide, glutathione reductase (GR) and NADPH in buffered solution, and monitors spectrophotometrically the initial rate of decrease of NADPH absorbance in consequence of its consumption by GR to reduce GSSG, formed upon reduction of $\mathrm{H}_{2} \mathrm{O}_{2}$ [110]. We found particularly useful the method originally described by Iwaoka and Tomoda [111], which uses thiophenol in place of GSH in the absence of any enzyme and co-factor, and directly monitors the initial rate of formation of diphenyl disulfide (eq. $24, \mathrm{R}=\mathrm{Ph}$ ) at $305 \mathrm{~nm}$, in the presence/absence of the test antioxidant, using diphenyl diselenide as reference antioxidant. Although the method affords a thiol peroxidase activity rather than true glutathione peroxidase, it is suitable for a variety of solvents and avoids complications due to interference of the test compound with GR or NADPH [110].

SOD-mimetic activity. Removal of superoxide by superoxide dismutase [EC 1.15.1.1] is an important preventive antioxidant process in biological systems and testing SOD-mimic antioxidants is a relevant need.

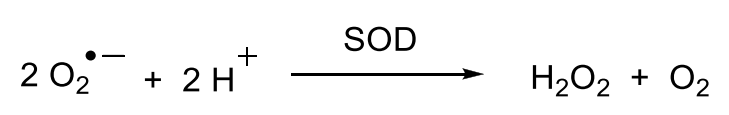

The most popular method uses xantine/xantine oxidase system at $\mathrm{pH} 7.8$ to produce at a constant rate superoxide, which is revealed by reaction with nitro blue tetrazolium (NBT assay) that is reduced to blue formazane, determined spectrophotometrically at $560 \mathrm{~nm}$. SOD-mimetic activity is determined from $\%$ inhibition of colour formation with respect of control experiments and expressed as IC50, the concentration that gives 50\% inhibition of NBT reduction [112]. Although popular, the main limit of this method is the inability to provide actual rate data for the dismutation of superoxide (the catalytic activity), furthermore it is prone to artifacts since the antioxidant could 
directly reduce NBT or inhibit xantine oxidase, thereby misestimating antioxidant performance.

Direct kinetic measurements of superoxide dismutation by pulse radiolysis or stopped-flow kinetics bring more valuable data [113] but require sophisticated equipment.

Additionally, SOD-mimetic activity can be investigated in cell-based assays, e.g. by assessing the survival of SOD-deficient yeasts (S. cerevisiae) [112]. Similar cell-based approaches have also been described for other enzyme-mimetic activities.

\section{Selection of the most appropriate testing method}

Most if not all the compounds from a chemical catalogue might show some remarkable activity when tested with some assay under some conditions. Unfortunately, the selection of the "best method" cannot be guided by its popularity in the scientific literature, which tends to favor simplicity over soundness and may become a self-supporting parameter.

On the basis of our previous discussion, inhibited autoxidation studies, initiated under controlled conditions by an azo-compound, should be regarded as the golden standard in antioxidants testing, because they distinctively provide information on the kinetics and stoichiometry of reaction with peroxyl radicals, as well as direct evidence of the ability of test compounds to act as antioxidants. A distinction needs to be made with regard of the testing needs. When pure molecules have to be tested as antioxidants or redox-active drugs, it is very important to achieve mechanistic insights that will enable drawing structure-activity relationships. In this case inhibited autoxidation studies are the most valuable. They should monitor the time course of reaction and/or focus on the kinetic aspects, while single-point measurements of secondary oxidation products might serve as preliminary tests. Similarly, methods based on competitive probe reaction or indirect methods based on persistent radicals should only be used for preliminary screening purposes. Whenever it is possible they should be used in kinetic mode, i.e. by monitoring the time evolution of the reaction; this is especially the case for DPPH test, which might provide valuable data. Due to their simplicity they might be useful to screen the antioxidant content of natural extracts or matrices of unknown 
composition. In this case, a clear distinction should be made between titrating the "potential antioxidant content" of the extract and achieving knowledge on the antioxidant activity or performance of those antioxidants. Titrations can conveniently be performed with indirect methods (e.g. DPPH or TEAC assays), however one should be aware that overestimation of antioxidant content is a likely possibility (e.g. by exchanging a highly oxidizable or reducing compound for a real antioxidant) [4] and those assays cannot replace full analytical characterization of the extract [114]. Assessing the antioxidant activity of extracts of unknown composition requires a kinetic investigation. Inhibited autoxidation studies can be very useful and should be preferred, however methods based on competitive probe reaction or indirect methods can be used as preliminary tests, but should be performed and analyzed in kinetic mode. Clearly kinetic studies referred to an extract possibly containing many active molecules can't provide real rate constants or stoichiometries of reaction, but they could provide apparent "specific" rate constants $(e . g$. rate constants per $\mathrm{mg} / \mathrm{mL}$ of extract) [88].

In no case assays based on single-point measurements should be used in structure-activity or mechanistic studies. It is the authors' opinion that indirect methods not involving organic radicals, such as those based on the reduction of transition metals, should not be used in antioxidant testing. They are unable to distinguish an antioxidant from any other reducing agent; therefore, beside the limitations noted for any indirect method, they are particularly prone to artefacts, often resulting in large overestimation of the antioxidant activity.

Specific testing needs might require specific additional studies not considered here $[115,116]$.

Furthermore, preventive antioxidant behavior can be investigated by metal binding studies $[99,100]$, or by kinetic studies aimed at mimicking the chemistry of glutathione peroxidase [110], or SOD and CAT, as discussed in the previous section.

None of the above assays can be expected to provide the biological activity of antioxidants, therefore cell-based assays could bring useful complementary information, upon careful 
consideration of their critical aspects. In general, however, no test is free of criticism or can be applied in a "blind" fashion.

\section{Declaration of Interest.}

Funding from the University of Bologna (NANOX project) FFBO123154, MIUR (Rome, Italy) PRIN 2010-2011 2010PFLRJR (PROxi project) and COST Action CM1201 (Biomimetic Radical Chemistry). Authors declare no competing financial interest.

\section{References}

[1] Ingold KU. Peroxyl radicals. Acc Chem Res. 1969; 2:1-9

[2] Valgimigli L, Pratt DA. Antioxidants in chemistry and biology. In: Chatgilialoglu C, Studer A, Editors. Encyclopedia of radicals in chemistry, biology and materials. Chirchester, UK: Wiley; 2012. Vol. 3, p 1623-1677.

[3] Ingold KU, Pratt DA. Advances in radical-trapping antioxidant chemistry in the 21st century: a kinetics and mechanisms perspective. Chem Rev. 2014;114:9022-9046

[4] Amorati R, Foti MC, Valgimigli L. Antioxidant activity of essential oils. J Agric Food Chem. 2013;61:10835-10847.

[5] Burton GW, Ingold KU. Vitamin E: Application of the principles of physical organic chemistry to the exploration of its structure and function. Acc Chem Res. 1986;19:194-201.

[6] Halliwell B, Gutteridge J. Free Radicals in Biology and Medicine, 4th ed.. Oxford: Oxford University Press; 2007.

[7] Niki E. Lipid antioxidants: how they may act in biological systems. Br J Cancer . 1987;55 Suppl. VIII:153-157.

[8] Valgimigli L, Iori R. Antioxidant and pro-oxidant capacities of ITCs. Environ Mol Mutagen. 2009;50:222-237. 
[9] Matera R, Gabbanini S, Berretti S, Amorati R, De Nicola GR, Iori R, Valgimigli L. Acylated anthocyanins from sprouts of Raphanus sativus cv. Sango: Isolation, structure elucidation and antioxidant activity. Food Chem. 2015; 166;397-406.

[10] Valgimigli L, Lucarini M, Pedulli GF, Ingold KU. Does beta-carotene really protect vitamin E from oxidation? J. Am. Chem. Soc. 1997;119:8095-8096

[11] Valgimigli L. Antioxidant supplementation in health promotion and modulation of aging: an overview. In: Watson RR and Preedy VR Editors. Bioactive Food as Dietary Interventions for the Aging Population. San Diego: Academic Press; 2013. p. 1-20.

[12] Gutteridge JMC, Halliwell B. Antioxidants: molecules, medicines, and myths. Biochem Biophys Res Comm. 2010;393:561-564.

[13] Valgimigli L, Sapone A, Canistro D, Broccoli M, Gatta L, Soleti A, Paolini M. Oxidative stress and aging: a non-invasive EPR investigation in human volunteers. Aging Clin Exp Res. DOI $10.1007 / \mathrm{s} 40520-014-0265-4$

[14] Canistro D, Boccia C, Falconi R, Bonamassa B, Valgimigli L, Vivarelli F, Soleti A, Genova ML, Lenaz G, Sapone A, Zaccanti F, Abdel-Rahman SZ, Paolini M. Redox-based flagging of the global network of oxidative stress greatly promotes longevity. J Gerontol A Biol Sci Med Sci. doi:10.1093/gerona/glu160.

[15] Roginsky V, Lissi EA. Review of methods to determine chain-breaking antioxidant activity in food. Food Chem. 2005;92:235-254.

[16] Niki E. Assessment of antioxidant capacity in vitro and in vivo. Free Radic Biol Med. 2010;49:503-515.

[17] Niki E, Noguchi N. Evaluation of antioxidant capacity. What capacity is being measured by which method? Life. 2000;50:323-329.

[18] Halliwell B, Whiteman M. Measuring reactive species and oxidative damage in vivo and in cell culture: how should you do it and what do the results mean? Brit J Pharmacol. 2004;142:231255. 
[19] Cheli F, Baldi A. Nutrition-based health: cell-based bioassays for food antioxidant activity evaluation. J Food Sci. 2011;76:R197-R205.

[20] López-Alarcón C, Denicola A. Evaluating the antioxidant capacity of natural products: A review on chemical and cellular-based assays. Anal. Chim. Acta. 2013;763:1-10.

[21] Liu RH, Finley J. Potential cell culture models for antioxidant research. J Agric Food Chem. 2005;53:4311-4314.

[22] Liu Z-Q. Chemical methods to evaluate antioxidant ability. Chem. Rev. 2010;110:5675-5691

[23] Huang D, Ou B, Prior RL. The chemistry behind antioxidant capacity assays. J. Agric. Food Chem. 2005;53:1841-1856

[24] Prior RL, Wu X, Schaich K. Standardized methods for the determination of antioxidant capacity and phenolics in foods and dietary supplements. J. Agric. Food Chem. 2005;53:4290-4302. [25] Amorati R, Valgimigli L. Modulation of the antioxidant activity of phenols by non-covalent interactions. Org Biomol Chem. 2012;10:4147-4158

[26] Halliwell B. The antioxidant paradox: less paradoxical now? Brit J Clin Pharmacol. 2012;75:637-644.

[27] Amorati R, Valgimigli L, Dinér P, Bakhtiari K, Saeedi, M, Engman L. Multi-faceted reactivity of alkyltellurophenols towards peroxyl radicals: catalytic antioxidant versus thioldepletion effect. Chem Eur J. 2013;19:7510-7522.

[28] Yamamoto K, Takahashi M, Niki E. Role of iron and ascorbic acid in the oxidation of methyl linoleate micelles. Chem Lett. 1987:1149-1152.

[29] Buxton GV, Greenstock CL, Helman WP, Ross AB. Critical review of rate constants for reactions of hydrated electrons, hydrogen atoms and hydroxyl radicals $(\mathrm{OH} / \cdot \mathrm{O}-$ in aqueous Solution. J Phys Chem Ref Data. 1988;17:513-886.

[30] Sawyer DT, Valentine JS. How super is superoxide? Acc Chem Res. 1981;14:393-400.

[31] Dickinson BC, Chang CJ. Chemistry and biology of reactive oxygen species in signaling or stress responses. Nat Chem Biol. 2011;7:504-511. 
[32] Haag WR, Yao CCD. Rate constants for reaction of hydroxyl radicals with several drinking water contaminants. Environ Sci Technol. 1992;26:1005-1013.

[33] Pignatello JJ, Oliveros E, MacKay A. Advanced oxidation processes for organic contaminant destruction based on the Fenton reaction and related chemistry. Critical Rev Environ Sci Technol. 2006;36:1-84.

[34] Hardwick TJ. The reactivity of hydrogen atoms in the liquid phase. III. The reaction with olefins. J Phys Chem. 1962;66, 291-295.

[35] Amorati R, Lynett PT, Valgimigli L, Pratt DA. The reaction of sulfenic acids with peroxyl radicals: insights into the radical trapping antioxidant activity of plant-derived thiosulfinates. Chem Eur J. 2012;18:6370-6379.

[36] Amorati R, Pedulli GF, Valgimigli L. Kinetic and thermodynamic aspects of the chainbreaking antioxidant activity of ascorbic acid derivatives in non-aqueous media. Org Biomol Chem. 2011;9:3792-3800.

[37] Lucarini M, Pedulli GF, Valgimigli L, Amorati R, Minisci F. Thermochemical and kinetic studies of a bisphenol antioxidant. J. Org. Chem. 2001;66:5456-5462.

[38] Amorati R, Pedulli GF, Cabrini L, Zambonin L, Landi L. Solvent and $\mathrm{pH}$ effects on the antioxidant activity of caffeic and other phenolic acids. J Agric Food Chem. 2006;54:2932-2937. [39] Hay KX, Waisundara VY, Timmins M, Ou B, Pappalardo K, McHale N, Huang D. Highthroughput quantitation of peroxyl radical scavenging capacity in bulk oils. J Agric Food Chem. 2006;54:5299-5305.

[40] Amorati R, Valgimigli L, Panzella L, Napolitano A, d'Ischia M.. 5-S-Lipoylhydroxytyrosol, a Multidefense Antioxidant Featuring a Solvent-Tunable Peroxyl Radical-Scavenging 3-Thio-1,2dihydroxybenzene Motif. J. Org. Chem. 2013;78:9857-9864

[41] Roschek Jr B, Tallman KA, Rector CL, Gillmore JG, Pratt DA, Punta C, Porter NA. Peroxyl radical clocks. J Org Chem. 2006;71:3527-3532. 
[42] Hanthorn JJ, Pratt DA. Peroxyesters as precursors to peroxyl radical clocks. J Org Chem. 2012;77:276-284.

[43] Spickett CM, Wiswedel I, Siems W, Zarkovic K, Zarkovic N. Advances in methods for the determination of biologically relevant lipid peroxidation products. Free Radical Res. 2010;44:11721202.

[44] Hanthorn JJ, Haidasz E, Gebhardt P, Pratt DA. A versatile fluorescence approach to kinetic studies of hydrocarbon autoxidations and their inhibition by radical-trapping antioxidants. Chem Commun. 2012;48:10141-10143.

[45] Foti M, Ruberto G. Kinetic solvent effects on phenolic antioxidants determined by spectrophotometric measurements. J Agric Food Chem. 2001;49:342-348.

[46] Kumar S, Johansson H, Engman L, Valgimigli L, Amorati R, Fumo M G, Pedulli GF. Regenerable chain-breaking 2,3-dihydrobenzo[b]selenophene-5-ol antioxidants. J Org Chem. $2007 ; 72: 2583-2595$.

[47] Bozin B, Mimica-Dukic N, Samojlik I, Jovinj E. Antimicrobial and antioxidant properties of rosemary and sage (Rosmarinus of $\mathrm{f}$ icinalis L. and Salvia officinalis L., Lamiaceae) essential oils. J Agric Food Chem. 2007;55:7879-7885.

[48] Teissedre PL, Waterhouse AL. Inhibition of oxidation of human low-density lipoproteins by phenolic substances in different essential oils varieties. J Agric Food Chem. 2000;48:3801-3805. [49] Matera R, Gabbanini S, Valvassori A, Triquigneaux M, Valgimigli L. Reactivity of (E)-4hydroxy-2-nonenal with fluorinatedphenylhydrazines: toward the efficient derivatization of an elusive key biomarker of lipid peroxidation. Eur J Org Chem. 2012;3841-3851.

[50] Viuda-Martos M, Navajas YR, Zapata ES, Fernández-López J, Pérez-Álvarez JA. Antioxidant activity of essential oils of five spice plants widely used in a Mediterranean diet. Flavour Fragr J. 2010;25:13-19.

[51] Bisby RH, Brooke R, Navaratnam S. Effect of antioxidant oxidation potential in the oxygen radical absorption capacity (ORAC) assay. Food Chem. 2008;108:1002-1007. 
[52] Cao G, Alessio HM, Culter R. Oxygen-radical absorbance capacity assay for antioxidants. Free Radic Biol Med. 1993;14:303-311.

[53] Ou B, Hampsch-Woodill M, Prior RL. Development and validation of an improved oxygen radical absorbance capacity assay using fluorescein as the fluorescent probe. J Agric Food Chem. 2001;49:4619-4626.

[54] Pompella A, Sies H, Wacker R, Brouns F, Grune T, Biesalski HK, Frank J. The use of total antioxidant capacity as surrogate marker for food quality and its effect on health is to be discouraged. Nutrition 2014;30:791-793.

[55] López-Alarcón C, Lissi E. A novel and simple ORAC methodology based on the interaction of pyrogallol red with peroxyl radicals. Free Radical Res. 2006;40:979-985.

[56] Sacchetti G, Maietti S, Muzzoli M, Scaglianti M, Manfredini S, Radice M, Bruni R. Comparative evaluation of 11 essential oils of different origin as functional antioxidants, antiradicals and antimicrobials in foods. Food Chem. 2005;91:621-632.

[57] Foti MC, Amorati R. Non-phenolic radical-trapping antioxidants. J Pharm Pharmacol 2009;61:1435-1448.

[58] Bortolomeazzi R, Sebastianutto N, Toniolo R, Pizzariello A. Comparative evaluation of the antioxidant capacity of smoke flavouring phenols by crocin bleaching inhibition, DPPH radical scavenging and oxidation potential. Food Chem. 2007;100:1481-1489.

[59] Kohri S, Fujii H, Oowada S, Endoh N, Sueishi Y, Kusakabe M, Shimmei M, Kotake Y. An oxygen radical absorbance capacity like assay that directly quantifies the antioxidant's scavenging capacity against AAPH-derived free radicals. Anal Biochem 2009;386:167-171.

[60] Amorati R, Pedulli GF, Pratt DA, Valgimigli L. TEMPO reacts with oxygen-centered radicals under acidic conditions. Chem Commun. 2010;46:5139-5141.

[61] Vladimirov YA, Proskurnina EV. Free radicals and cell chemiluminescence. BiochemistryMoscow 2009;74:1545-1566. 
[62] Lissi E, Salim-Hanna M, Pascual C, del Castillo MD. Evaluation of total antioxidant potential (TRAP) and total antioxidant reactivity from luminol-enhanced chemiluminescence measurements, Free Radic Biol Med. 1995;18:153-158.

[63] Winston GW, Regoli F, Dugas AJ, Fong JH, Blanchard KA. A rapid gas chromatographic assay for determining oxyradical scavenging capacity of antioxidants and biological fluids. Free Radic Biol Med. 1998;24:480-493.

[64] Wojtunik KA, Ciesla LM, Waksmundzka-Hajnos M. Model studies on the antioxidant activity of common terpenoid constituents of essential oils by means of the 2, 2-diphenyl-1picrylhydrazyl method. J Agric Food Chem. 2014;62:9088-9094.

[65] Ionita P. Is DPPH stable free radical a good scavenger for oxygen active species? Chem Pap. 2005;59:11-16.

[66] Amorati R, Menichetti S, Viglianisi C, Foti MC. Proton-electron transfer pathways in the reactions of peroxyl and dpph radicals with hydrogen-bonded phenols. Chem Commun. 2012;48:11904-11906.

[67] Musialik M, Kuzmicz R, Pawłowski TS, Litwinienko G. Acidity of hydroxyl groups: an overlooked influence on antiradical properties of flavonoids. J Org Chem. 2009;74:2699-2709. [68] Foti MC, Amorati R, Pedulli GF, Daquino C, Pratt DA, Ingold KU. Influence of "remote" intramolecular hydrogen bonds on the stabilities of phenoxyl radicals and benzyl cations. J. Org. Chem. 2010;75:4434-4440.

[69] Litwinienko G, Ingold KU. Abnormal solvent effects on hydrogen atom abstractions. 1. The reactions of phenols with 2,2-diphenyl-1-picrylhydrazyl (dpph•) in alcohols. J Org Chem. 2003;68:3433-3438.

[70] Amorati R, Menichetti S, Mileo E, Pedulli GF, Viglianisi C. Hydrogen-atom transfer reactions from ortho-alkoxy-substituted phenols: an experimental approach. Chem Eur J. 2009;15:4402-4410. 
[71] Re R, Pellegrini N, Proteggente A, Pannala A, Yang M, Rice-Evans C. Antioxidant activity applying an improved ABTS radical cation decolorization assay. Free Radic Biol Med 1999;26:1231-1237.

[72] Tian X, Schaich KM. Effects of molecular structure on kinetics and dynamics of the Trolox Equivalent Antioxidant Capacity assay with ABTS $^{+\bullet}$. J Agric Food Chem. 2013;61:5511-5519.

[73] Perez-Jimenez J, Saura-Calixto F. Anti-oxidant capacity of dietary polyphenols determined by ABTS assay: a kinetic expression of the results. Int J Food Sci Technol 2008;48:185-191.

[74] Tai A, Sawano T, Yazama F, Ito H. Evaluation of antioxidant activity of vanillin by using multiple antioxidant assays. BBA-Gen Subjects 2011;1810:170-177.

[75] Benzie IFF, Strain JJ. The ferric reducing ability of plasma (FRAP) as a measure of 'antioxidant power': the FRAP assay. Anal Biochem. 1996;239:70-76.

[76] Apak R, Güçlü K, Özyürek M, Karademir SE. Novel total antioxidant capacity index for dietary polyphenols and vitamins $\mathrm{C}$ and $\mathrm{E}$, using their cupric ion reducing capability in the presence of neocuproine: CUPRAC method. J Agric Food Chem 2004;52:7970-7981.

[77] Scampicchio M, Wang J, Blasco AJ, Sanchez Arribas A, Mannino S, Escarpa A. Nanoparticle-based assays of antioxidant activity. Anal Chem. 2006;78:2060-2063.

[78] Sharpe E, Frasco T, Andreescu D, Andreescu S. Portable ceria nanoparticle-based assay for rapid detection of food antioxidants (NanoCerac). Analyst 2013;138:249-262.

[79] Doba T, Burton GW, Ingold KU. Antioxidant and co-antioxidant activity of vitamin C. The effect of vitamin $\mathrm{C}$, either alone or in the presence of vitamin $\mathrm{E}$ or a water-soluble vitamin $\mathrm{E}$ analogue, upon the peroxidation of aqueous multilamellar phospholipid liposomes. Biochim Biophys Acta. 1985;835:298-303.

[80] Halliwell B. Free radicals and antioxidants - quo vadis? Trends Pharmacol. Sci. 2011;32:125130

[81] Halliwell B. Hypothesis—oxidative stress in cell culture: an under-appreciated problem? FEBS Lett. 2003;540:3-6 
[82] Wolfe KL, Liu RH. Cellular antioxidant activity (CAA) assay for assessing antioxidants, foods, and dietary supplements. J Agric Food Chem. 2007;55:8896-907.

[83] Honzel D, Carter SG, Redman KA, Schauss AG, Endres JR, Jensen GS. Comparison of chemical and cell-based antioxidant methods for evaluation of foods and natural products: generating multifaceted data by parallel testing using erythrocytes and polymorphonuclear cells. J Agric Food Chem. 2008;56:8319-25.

[84] Blasa M, Angelino D, Gennari L, Ninfali P. The cellular antioxidant activity in red blood cells (CAA-RBC): a new approach to bioavailability and synergy of phytochemicals and botanical extracts. Food Chem. 2011;125:685-91.

[85] Halliwell B. Free radicals and antioxidants: updating a personal view. Nutr. Rev. 2012;70:257-265

[86] Zhang D, Xie L, Wei Y, Liu Y, Jia G, Zhou F, Ji B. Development of a cell-based antioxidant activity assay using dietary fatty acid as oxidative stressor. Food Chem. 2013;141:347-356 [87] Papi A, Orlandi M, Bartolini G, Barillari J, Iori R, Paolini M, Ferroni F, Fumo, MG, Pedulli GF, Valgimigli L. Cytotoxic and antioxidant activity of 4-methylthio-3-butenyl isothiocyanate from Raphanus sativus L. (Kaiware Daikon) sprouts. J Agric Food Chem. 2008;56:875-883.

[88] Barillari J, Iori R, Papi A, Orlandi M, Bartolini G, Gabbanini S, Pedulli GF, Valgimigli L. Kaiware Daikon (Raphanus sativus L.) extract: a naturally multipotent chemopreventiveaAgent. J Agric Food Chem. 2008;56:7823-7830

[89] Marchesi E, Rota C, Fann YC, Chignell CF, Mason RP. Photoreduction of the fluorescent dye 2'-7'-dicholorofluorescein: a spin trapping and direct electron spin resonance study with implications for oxidative stress measurments. Free Radic Biol. Med. 1999;26:148-161. [90] Bonini, MG, Rota C, Tomasi A, Mason RP. The oxidation of 2',7'-dichlorofluorescin to reactive oxygen species: A self-fulfilling prophesy? Free Radic Biol Med. 2006;40:968-975. 
[91] Krumova K, Greene LE, Cosa G. Fluorogenic $\alpha$-Tocopherol analogue for monitoring the antioxidant status within the inner mitochondrial membrane of live cells. J Am Chem Soc. $2013 ; 135: 17135-17143$

[92] Valgimigli L, Pedulli GF, Paolini M. Measurment of oxidative stress by EPR radical-probe technique. Free Radic Biol. Med. 2001;31:708-916.

[93] Valgimigli L, Valgimigli M, Gaiani S, Pedulli GF, Bolondi L. Measurement of oxidative stress in human liver by EPR spin-probe technique. Free Radical Res. 2000;33:167-178.

[94] Valgimigli M, Valgimigli L, Trerè D, Gaiani S, Pedulli GF, Gramantieri L, Bolondi L. Oxidative stress EPR measurement inhHuman liver by radical-probe technique. Correlation with etiology, histology and cell proliferation. Free Radic Res. 2002;36:939-948.

[95] Paolini M, Valgimigli L, Marchesi E, Trespidi S, Pedulli GF. Taking EPR “snapshots” of the oxidative stress status in human blood. Free Radical Res. 2003;37:503-508.

[96] Zhang YS, Talalay P, Cho CG, Posner GH. A major inducer of anticarcinogenic protective enzymes from broccoli-isolation and elucidation of structure. Proc Natl Acad Sci USA. 1992;89:2399-2403

[97] Manjamalai, A.; Berlin Grace, V. M. Antioxidant activity of essential oils from Wedelia chinensis (Osbeck) in vitro and in vivo lung cancer bearing C57BL/6 mice. Asian Pac J Cancer Prev. 2012;13:3065-3071.

[98] Prawan A, Keum YS, Khor TO, Yu S, Nair S, Li W, Hu L, Kong AN. Structural influence of isothiocyanates on the antioxidant response element (ARE)-mediated heme oxygenase-1 (HO-1) expression. Pharm Res. 2008;25:836-844.

[99] Antunes F, Barclay LRC, Ingold KU, King M, Norris JQ, Scaiano JC, Xi F. On the antioxidant activity of melatonin. Free Radic. Biol. Med. 1999;26:117-128.

[100] Bolognesi ML, Cavalli A, Valgimigli L, Bartolini M, Rosini M, Andrisano V, Recanatini M, Melchiorre C. Multi-target-directed drug design strategy: from a dual binding site 
acetylcholinesterase inhibitor to a trifunctional compound against Alzheimer's disease. J. Med. Chem. 2007;50:6446-6449

[101] Barillari J, Canistro D, Paolini M, Ferroni F, Pedulli GF, Iori R, Valgimigli L. Direct antioxidant activity of purified glucoerucin, the dietary secondary metabolite contained in Rocket (Eruca sativa Mill.) seeds and sprouts. J Agric Food Chem. 2005;53:2475-2482.

[102] Beers RF, Jr, Sizer IW. A spectrophotometric method for measuring the breakdown of hydrogen peroxide by catalase. J Biol Chem. 1952;195:133-140.

[103] Li Y, Schelhorn HE. Rapid kinetic microassay for catalase activity. J Biomol Tech. 2007;18:185-187.

[104] Zhou M, Diwu Z, Panchuk-Voloshina N, Haugland RP A Stable nonfluorescent derivative of resorufin for the fluorometric determination of trace hydrogen peroxide: applications in detecting the activity of phagocyte NADPH oxidase and other oxidases. Anal Biochem. 1997;253:162-168. [105] Votyakova TV, Reynolds IJ. Detection of hydrogen peroxide with Amplex Red: interference by NADH and reduced glutathione auto-oxidation. Arch Biochem Biophys. 2004;431:138-144. [106] Del Río LA, Ortega MG, López AL, Gorgé JL. A more sensitive modification of the catalase assay with the Clark oxygen electrode. Application to the kinetic study of the pea leaf enzyme. Anal Biochem. 1977;80:409-15

[107] Escobar L, Salvador C, Contreras M, Escamilla JE. On the application of the Clark oxygen electrode to the study of enzyme kinetics in apolar solvents: the catalase reaction. Anal Biochem. 1990;184:139-44.

[108] Iwase T, Tajima A, Sugimoto S, Okuda K, Hironaka I, Kamata Y, Takada K, Mizunoe Y. A simple assay for measuring catalase activity: a visual approach. Sci Rep. 2013;3:3081.

[109] Mugesh G, Singh B. Synthetic organoselenium compounds as antioxidants: glutathione peroxidase activity. Chem Soc Rev. 2000;29:347-357.

[110] Kumar S, Engman L, Valgimigli L, Amorati R, Fumo MG, Pedulli GF. Antioxidant profile of ethoxyquin and some of its S, Se, and Te analogues. J Org Chem. 2007;72:6046-6055 
[111] Iwaoka M, Tomoda S. A model study on the effect of an amino group on the antioxidant activity of glutathione peroxidase. J Am Chem Soc. 1994;116:2557-2561.

[112] Gonzalez-Alvarez M, Alzuet G, Borrás J, del Castillo Agudo L, García-Granda S,MontejoBernardo JM. Comparison of protective effects against reactive oxygen species of mononuclear and dinuclear $\mathrm{Cu}(\mathrm{II})$ complexes with $\mathrm{N}$-substituted benzothiazolesulfonamides. Inorg Chem. 2005;44:9424-9433.

[113] Riley DP. Functional mimics of superoxide dismutase enzymes as therapeutic agents. Chem Rev. 1999;99:2573-2587

[114] Matera R, Gabbanini S, De Nicola GR, Iori R, Petrillo G, Valgimigli L. Identification and analysis of isothiocyanates and new acylated anthocyanins in the juice of Raphanus sativus cv. Sango sprouts. Food Chem. 2012;133:563-572

[115] Amorati R, Lynett PT, Valgimigli L, Pratt DA. The reaction of sulfenic acids with peroxyl radicals: insights into the radical-trapping antioxidant activity of plant-derived thiosulfinates. Chem Eur J. 2012;18:6370-6379

[116] Hanthorn JJ, Amorati A, Valgimigli L, Pratt DA. The reactivity of air-stable pyridine-and pyrimidine-containing diarylamine antioxidants. J Org Chem. 2012;77:6895-6907 
Table 1. Rate constants for the reaction of three phenolic antioxidants with DPPH and with ROO• radicals, showing the abnormal increase of $k_{\mathrm{DPPH}}$ in a protic solvent. ${ }^{\mathrm{a}}$

\begin{tabular}{|l|l|l|l|l|}
\hline \multicolumn{1}{|c|}{$2,6-{ }^{\mathrm{t}} \mathrm{Bu}_{2}-\mathrm{PhOH}$} & \multicolumn{3}{|c|}{$k_{\mathrm{DPPH}} / \mathrm{M}^{-1} \mathrm{~s}^{-1}$} & $k_{\mathrm{ROO}} / \mathrm{M}^{-1} \mathrm{~s}^{-1}$ \\
& heptane & $\mathrm{MeCN}$ & $\mathrm{MeOH}$ & $\mathrm{PhCl}$ \\
\hline $4-\mathrm{OMe}$ & 22.6 & 1.5 & 3.9 & $1.1 \times 10^{5}$ \\
\hline $4-\mathrm{Me}$ & 1.1 & 0.09 & 3.7 & $1.4 \times 10^{4}$ \\
\hline $4-\mathrm{CN}$ & 0.022 & 0.05 & 16 & $9.9 \times 10^{2}$ \\
\hline
\end{tabular}

a) Data from references 67 and 68 . 


\section{Legend to figures and schemes}

Scheme 1. Simplified chain-reaction of autoxidation for a substrate RH (e.g. linoleic acid) and mode of interferece by direct antioxidants.

Scheme 2. Simplified distribution of primary and secondary products from autoxidation of linoleic acid. XH represents a H-atom donor, be it an antioxidant or linoleic acid itself.

Scheme 3. Chemical structure of some oxidation probes.

Scheme 4. Mechanism that leads to light emission after the reaction of luminol with radicals.

Scheme 5. Mechanisms for the reaction of phenols with radicals, formal hydrogen atom transfer (HAT) and sequential proton-loss electron transfer (SPLET), in a H-bond accepting solvent.

Figure 1. Typical oxygen consumption plot obtained during the autoxidation of styrene (A) in the absence of antioxidants, (B) in the presence of a very good antioxidant, $(\mathrm{C})$ in the presence of a weak antioxidant. Upon kinetic treatment the plots can provide rate constants and stoichiometry.

Figure 2. Simulation of the decrease of the fluorescence and calculation of the differential area under the curve $(\triangle \mathrm{AUC})$ during the ORAC assay in the absence and in the presence of three antioxidants (AH1, $\mathrm{AH} 2, \mathrm{AH} 3)$ having different rate constants $\left(k_{\mathrm{ROO}}\right)$ and stoichiometries $(n)$ for the reaction with peroxyl radicals.

Figure 3. Simulation of the decrease of absorbance of DPPH• and calculation of the percent DPPH• quenching at three reaction times $\left(\mathrm{t}_{1}, \mathrm{t}_{2}, \mathrm{t}_{3}\right)$ in the presence of three antioxidants $(\mathrm{AH} 1, \mathrm{AH} 2, \mathrm{AH} 3)$ having different rate constants $\left(k_{\mathrm{DPPH}}\right)$ and stoichiometries $(n)$ for the reaction with DPPH $\bullet$ 


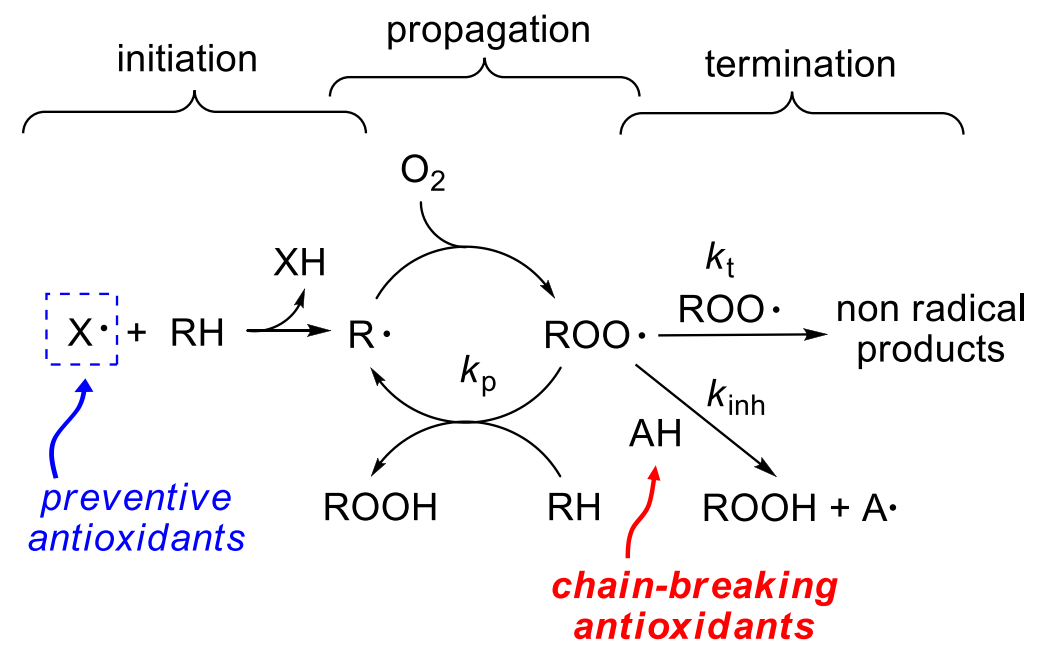

Scheme 1. Simplified chain-reaction of autoxidation for a substrate RH (e.g. linoleic acid) and mode of interferece by direct antioxidants.
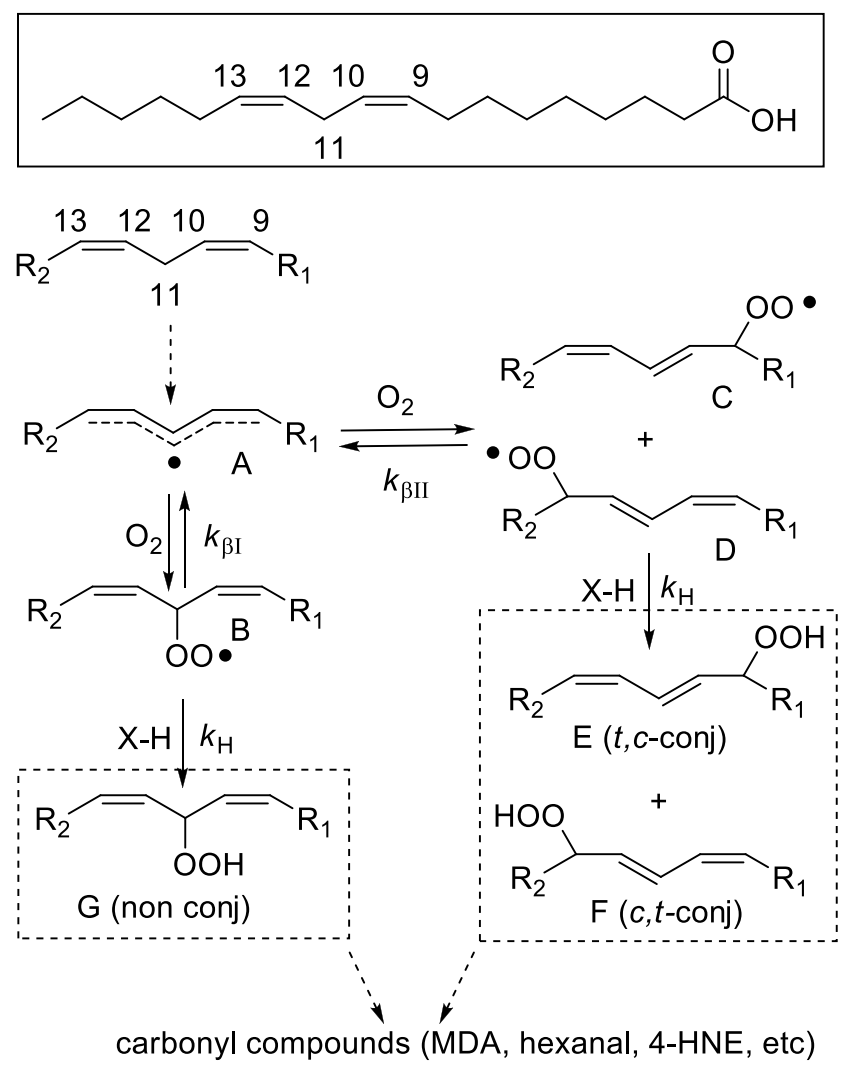

Scheme 2. Simplified distribution of primary and secondary products from autoxidation of linoleic acid. XH represents a H-atom donor, be it an antioxidant or linoleic acid itself. 
<smiles>O=C([O-])c1ccccc1-c1c2ccc(=O)cc-2oc2cc(O)ccc12</smiles>

fluorescein<smiles>O=c1ccc2c(-c3ccccc3S(=O)(=O)[O-])c3ccc(O)c(O)c3oc-2c1O</smiles>

pyrogallol red<smiles>OC[C@H]1CC[C@@H](O)[C@H](O)[C@@H]1O</smiles><smiles>CO</smiles>

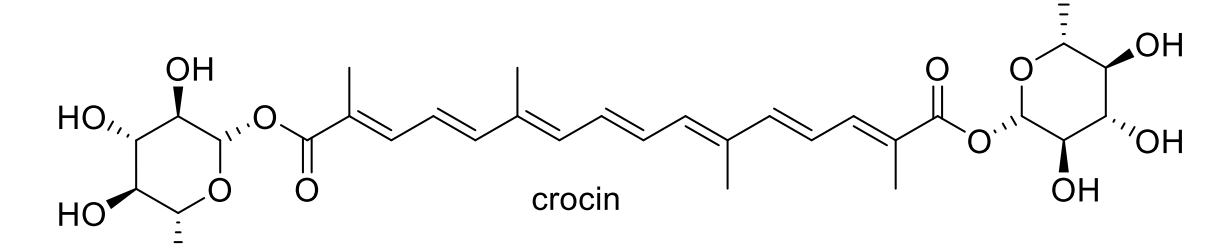<smiles>CO[C@H]1C[C@H](CO)[C@@H](O)[C@H](O)[C@H]1O</smiles>

Scheme 3. Chemical structure of some oxidation probes.

$\mathrm{LH}_{2}$<smiles>Nc1cccc2c(=O)[nH][nH]c(=O)c12</smiles>

$\mathrm{LH}^{\cdot}$<smiles></smiles>

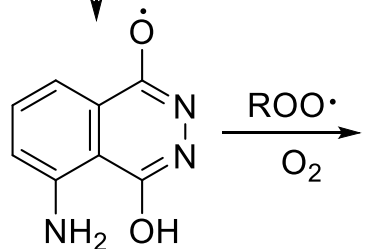<smiles>Nc1cccc2c1=C(O)OOC=2O</smiles>
$+\mathrm{N}_{2}$<smiles>COC(=O)c1cccc(N)c1C(=O)O</smiles>

Scheme 4. Mechanism that leads to light emission after the reaction of luminol with radicals. 


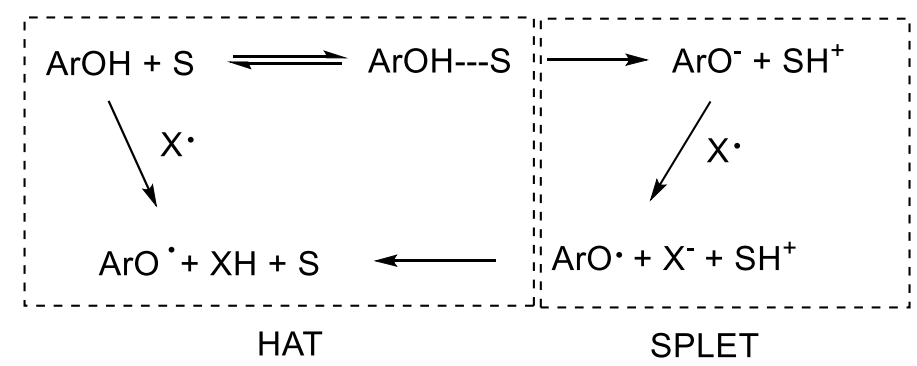

Scheme 5. Mechanisms for the reaction of phenols with radicals, formal hydrogen atom transfer (HAT) and sequential proton-loss electron transfer (SPLET), in a H-bond accepting solvent.

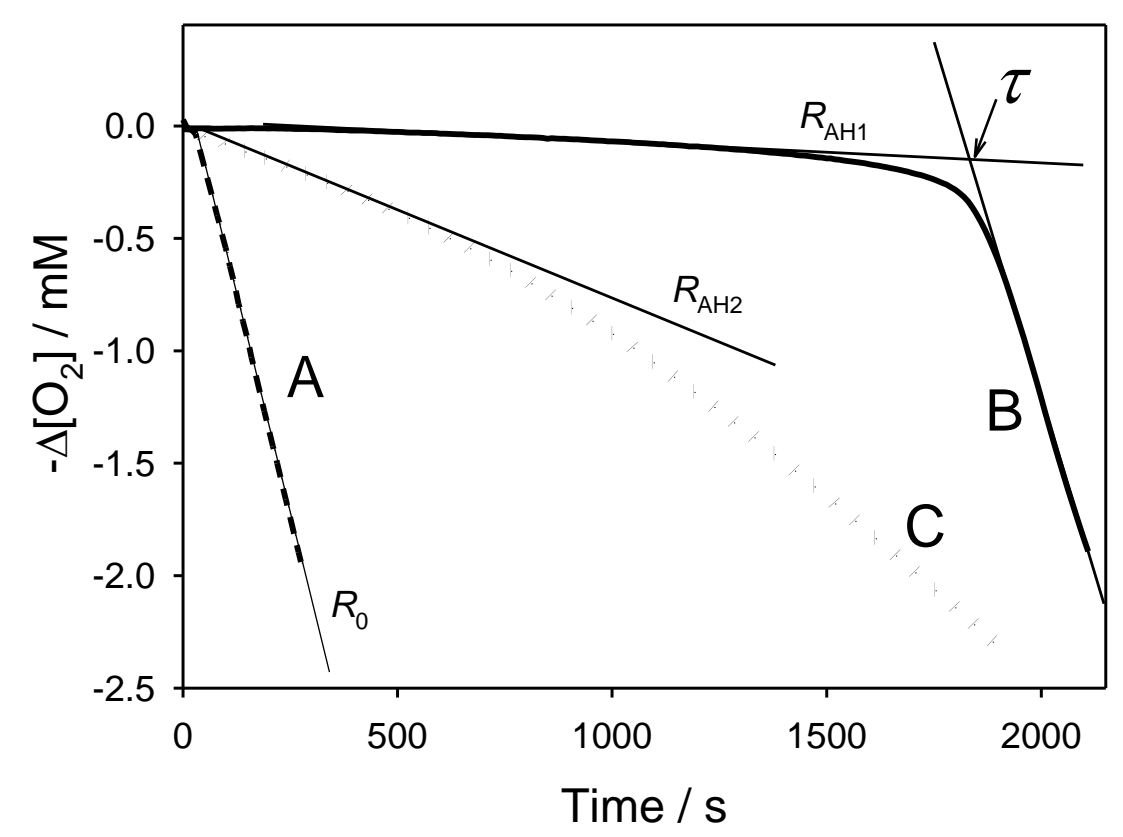

Figure 1. Typical oxygen consumption plot obtained during the autoxidation of styrene (A) in the absence of antioxidants, (B) in the presence of a very good antioxidant, $(\mathrm{C})$ in the presence of a weak antioxidant. Upon kinetic treatment the plots can provide rate constants and stoichiometry. 


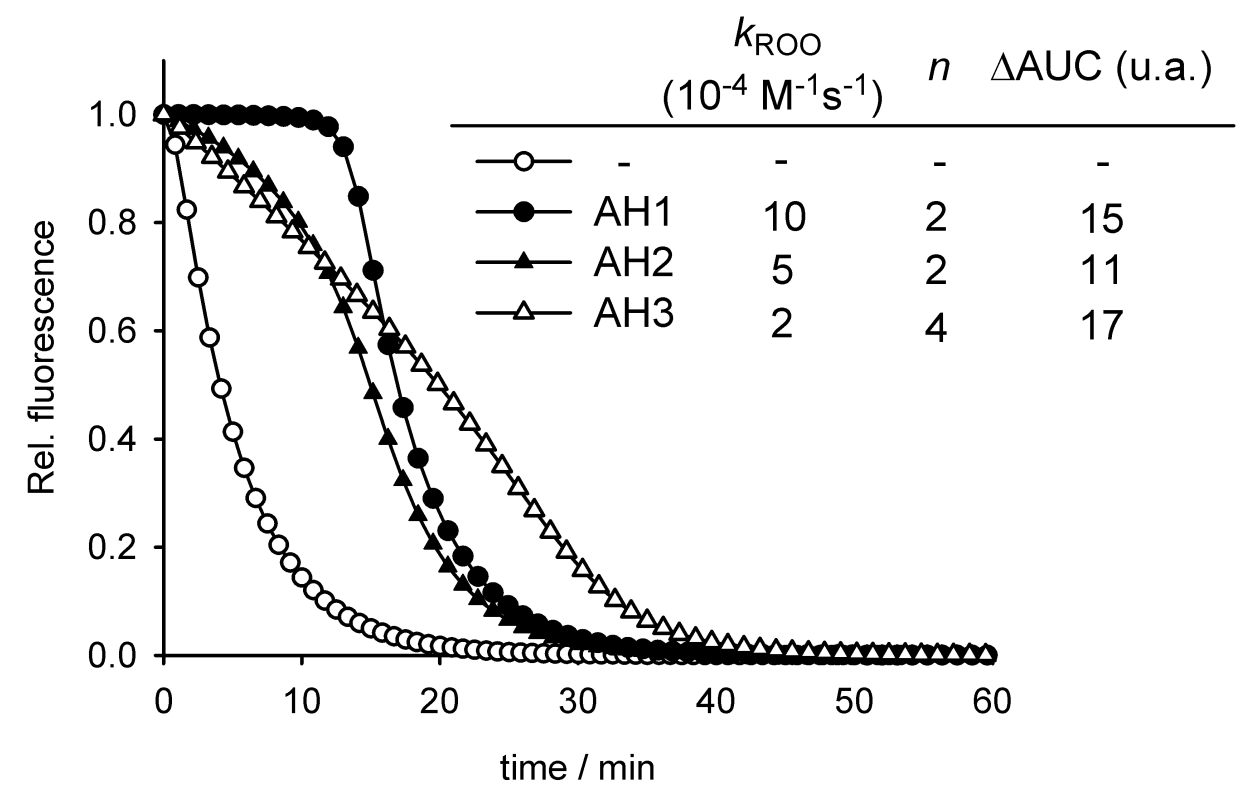

Figure 2. Simulation of the decrease of the fluorescence and calculation of the differential area under the curve ( $\triangle \mathrm{AUC}$ ) during the ORAC assay in the absence and in the presence of three antioxidants $(\mathrm{AH} 1, \mathrm{AH} 2, \mathrm{AH} 3)$ having different rate constants $\left(k_{\mathrm{ROO}}\right)$ and stoichiometries $(n)$ for the reaction with peroxyl radicals.

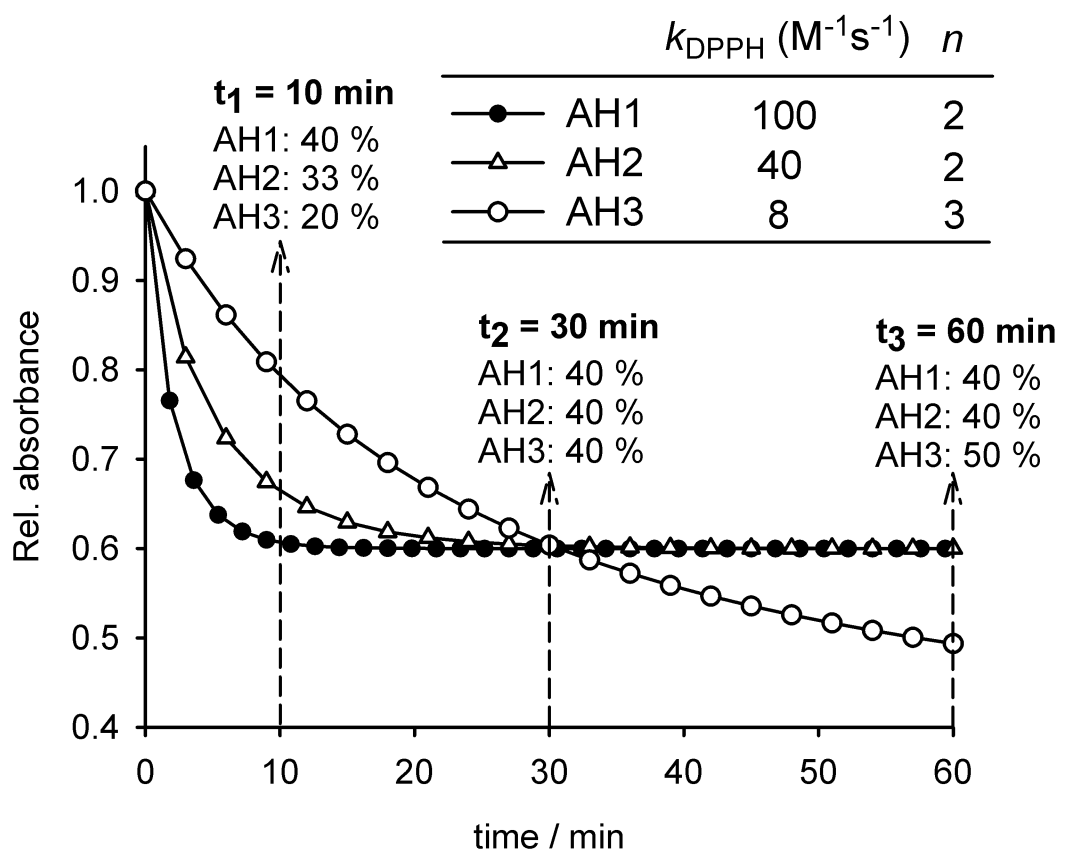

Figure 3. Simulation of the decrease of absorbance of DPPH• and calculation of the percent DPPH• quenching at three reaction times $\left(t_{1}, t_{2}, t_{3}\right)$ in the presence of three antioxidants $(\mathrm{AH} 1, \mathrm{AH} 2, \mathrm{AH} 3)$ having different rate constants $\left(k_{\mathrm{DPPH}}\right)$ and stoichiometries $(n)$ for the reaction with DPPH• 